\title{
A Well-posed and Stable Stochastic Galerkin Formulation of the Incompressible Navier-Stokes Equations with Random Data
}

\author{
Per Pettersson ${ }^{\mathrm{a}, *}$, Jan Nordström ${ }^{\mathrm{b}}$, Alireza Doostan ${ }^{\mathrm{c}}$ \\ ${ }^{a}$ Uni Research, N-5007 Bergen, Norway. \\ ${ }^{b}$ Department of Mathematics, Computational Mathematics, Linköping University, \\ SE-58183 Linköping, Sweden. \\ ${ }^{c}$ Aerospace Engineering Sciences, University of Colorado Boulder, CO 80309, USA.
}

\begin{abstract}
We present a well-posed stochastic Galerkin formulation of the incompressible Navier-Stokes equations with uncertainty in model parameters or the initial and boundary conditions. The stochastic Galerkin method involves representation of the solution through generalized polynomial chaos expansion and projection of the governing equations onto stochastic basis functions, resulting in an extended system of equations. A relatively low-order generalized polynomial chaos expansion is sufficient to capture the stochastic solution for the problem considered.

We derive boundary conditions for the continuous form of the stochastic Galerkin formulation of the velocity and pressure equations. The resulting problem formulation leads to an energy estimate for the divergence. With suitable boundary data on the pressure and velocity, the energy estimate implies zero divergence of the velocity field.

Based on the analysis of the continuous equations, we present a semidiscretized system where the spatial derivatives are approximated using finite difference operators with a summation-by-parts property. With a suitable choice of dissipative boundary conditions imposed weakly through penalty terms, the semi-discrete scheme is shown to be stable. Numerical experi-
\end{abstract}

\footnotetext{
*Corresponding author

Email addresses: per.pettersson@uib.no (Per Pettersson), jan.nordstrom@liu.se (Jan Nordström), alireza.doostan@colorado.edu (Alireza Doostan)
}

Preprint submitted to Journal of Computational Physics

November 11, 2015

(C) 2015. This manuscript version is made available under the Elsevier user license http://www.elsevier.com/open-access/userlicense/1.0/ 
ments in the laminar flow regime corroborate the theoretical results and we obtain high-order accurate results for the solution variables and the velocity divergence converges to zero as the mesh is refined.

Keywords: Uncertainty quantification, Incompressible Navier-Stokes equations, Summation-by-parts operators, Stochastic Galerkin method, Boundary conditions

\section{Introduction}

Complex fluid flow problems are frequently dependent on model parameters that rely on approximations. Accurate and reliable numerical simulations therefore require that we properly account for uncertainties in parameters, and in initial and boundary conditions. Forward uncertainty quantification deals with systematic and quantitative means of accounting for input uncertainty and methods to propagate it through the numerical model of interest. This process in turn requires numerical methods that produce accurate solutions under uncertainty [1].

A framework for efficient representation and propagation of uncertainty is offered by polynomial chaos methods, e.g., the stochastic Galerkin method where the governing equations are projected onto stochastic basis functions $[2,3]$. The aim of this paper is to present and investigate a new stochastic Galerkin formulation of the incompressible Navier-Stokes equations and a corresponding finite difference formulation. We are mainly interested in the case of uncertainty in the viscosity, but provide a framework that may include uncertainty in other model parameters as well as uncertainty in the initial and boundary conditions.

In the numerical computations, the governing equations are approximated within the order of accuracy of the numerical method. Neither the divergence relation nor the momentum equations are satisfied exactly on a finite computational grid, but we will derive a method that leads to high-order convergence in all solution variables, as well as in the divergence itself. The velocity-pressure formulation of the governing equations includes a pressure equation that constitutes the source terms in an advection-diffusion equation for the divergence. Setting these source terms equal to zero by solving the pressure equation, the advection-diffusion equation for the divergence is enforced implicitly. In combination with suitable boundary conditions, although the advection-diffusion equation is never explicitly solved, the di- 
vergence of the velocity is converging to zero with the order of the scheme used for the velocity-pressure formulation.

The stochastic Galerkin formulation introduces an error due to the truncated generalized polynomial chaos representation of the problem, and with a low-order stochastic approximation, this error may dominate the discretization error in both the solution variables and the divergence. However, even in the absence of stochastic truncation error it makes sense to sacrifice exact zero divergence for a provably stable solution of high order of accuracy where the error in the solution variables and the divergence can be made arbitrarily small through mesh refinement.

The incompressible Navier-Stokes equations with uncertain viscosity was previously investigated in [4], where a stochastic Galerkin formulation was presented and implemented with a fractional step method resulting in an advection-diffusion problem decoupled from the solution of the pressure and divergence system. The method was generalized to the weakly compressible Navier-Stokes equations and random process input uncertainty in [5]. A similar stochastic Galerkin method for the incompressible Navier-Stokes equations was devised in [6], and evaluated for different uncertain boundary conditions. Efficient representation of uncertainty was ensured by the choice of stochastic basis, i.e., orthogonal polynomials with the inner product weight function matching the probability density function of the boundary conditions. The stochastic Galerkin formulation of the incompressible Navier-Stokes equations was also employed for fluid-structure interaction to study vortex-induced vibrations in [7], and for flow under stochastic nonBoussinesq conditions in [8].

The purpose of this work is not a direct comparison with the previous incompressible Navier-Stokes stochastic Galerkin solvers listed above; that is the goal of a future investigation. Instead, we focus on the development of a new stochastic Galerkin solver and perform detailed analysis to prove relevant properties of the continous formulation and the proposed numerical method. The stochastic Galerkin projection results in an extended system of equations that is dependent on both the sources of uncertainty and the stochastic representation. In order to design general, robust and stable numerical schemes that can handle different forms of uncertainty and different stochastic representations, we use finite-difference operators with a summation-by-parts property $[9,10]$. This allows for the design of methods that are provably time stable [11] in combination with boundary conditions that are imposed weakly, i.e., through discrete penalty terms that vanish with spatial mesh 
refinement [12].

The analysis of well-posedness and stability for the stochastic Galerkin formulation presented in this paper extends the analysis presented for the deterministic incompressible Navier-Stokes problem in [13]. In that analysis, the condition of exact enforcement of the incompressibility condition was relaxed in order to gain overall stability and accuracy of the numerical method. We provide a stochastic Galerkin formulation that leads to an energy estimate for the velocity divergence. In combination with certain data requirements, this estimate shows that the zero divergence condition indeed is satisfied. Finally, by mimicking that procedure for the semi-discretized problem, we can prove stability and high-order convergence. The result is a stable high-order method that handle stochastic, non-periodic and time-dependent boundary conditions for the incompressible Navier-Stokes equations.

To appreciate both the similarities and the differences between the deterministic and the stochastic Galerkin formulation, we reproduce some of the results from [13] along with new results. The deterministic analysis serves as a roadmap for the stochastic Galerkin analysis, and the analysis of the continuous problem serves as a guide for the analysis of the semi-discrete problem. By proceeding step-wise, from continuous to semi-discrete formulation and from deterministic to stochastic Galerkin formulation, we derive a numerical method for the solution of the stochastic problem that may otherwise be difficult to derive.

To facilitate the reading, the main contributions of this work are summarized below.

- Analysis of well-posedness with derivation of proper boundary conditions for a stochastic Galerkin formulation of the incompressible NavierStokes equations.

- A roadmap for the non-trivial analysis of the semi-discrete stochastic Galerkin problem based on the analysis of the continuous and the deterministic problems.

- Derivation of numerical method that is proven to be time-stable for time-dependent and non-periodic stochastic boundary conditions.

- Zero divergence is imposed to the order of accuracy of the numerical method for the stochastic Galerkin problem. The result is high-order 
accurate in all variables, including the divergence. The method is efficient compared to standard methods that sacrifice the field variable accuracy to obtain exactly zero divergence.

- Numerical results that corroborate the theoretical findings and demonstrate high-order convergence for the solution variables and the velocity divergence.

In Section 2, we present the generalized polynomial chaos framework, and the stochastic Galerkin incompressible Navier-Stokes equations are presented in Section 3. The continuous formulation is analyzed in Section 4. The deterministic analysis is reproduced from [13], followed by analysis of wellposedness for the stochastic Galerkin formulation. The continuous analysis provides boundary conditions and a recipe for the analysis of stability of the spatially discretized problem in Section 5. Numerical results are presented in Section 6 for a Taylor vortex and a Poiseuille flow problem with stochastic viscosity. Finally, conclusions are discussed in Section 7.

\section{Representation of Uncertainty}

Following the seminal work in $[2,14]$, we represent stochastic functions by using the polynomial chaos framework. To this end, consider a probability space $(\Omega, \mathcal{F}, \mathcal{P})$ with the set of elementary events $\Omega$ and probability measure $\mathcal{P}$ defined on the $\sigma$-algebra $\mathcal{F}$. Let $\boldsymbol{\xi}=\left(\xi_{1}, \ldots, \xi_{d}\right)^{T}$ be a $d$-dimensional independent random vector defined on this space. The inner product between two stochastic functions $f(\boldsymbol{\xi})$ and $g(\boldsymbol{\xi})$ is defined by the expected value of their product

$$
\langle f, g\rangle \equiv\langle f g\rangle=\int_{\Omega} f(\boldsymbol{\xi}) g(\boldsymbol{\xi}) d \mathcal{P}(\boldsymbol{\xi}) .
$$

Consider a generalized Polynomial Chaos (gPC) basis $\left\{\psi_{i}(\boldsymbol{\xi})\right\}_{i=1}^{\infty}$ spanning the space of second order (i.e. finite variance) random processes on this probability space. Possible choices of basis functions include the orthogonal polynomials from the Askey scheme [15] and, for functions with non-smooth behavior, stochastic multiwavelets [16]. Multi-dimensional basis functions are generated by tensor products of one-dimensional basis functions. The subsequent analysis in this paper is valid for all these stochastic basis functions. The basis functions, denoted by $\psi_{i}$, are assumed to be orthonormal with respect to the inner product $\langle\cdot, \cdot\rangle$ with the measure $\mathcal{P}$, i.e., they satisfy

$$
\left\langle\psi_{i}, \psi_{j}\right\rangle=\delta_{i j}, \quad i, j \in \mathbb{N},
$$


where $\delta_{i j}$ is the Kronecker delta. Any second order random field $u(\mathbf{x}, t, \boldsymbol{\xi})$ (typically the solution of a partial differential equation) can be expressed as a gPC expansion in the form

$$
u(\mathbf{x}, t, \boldsymbol{\xi})=\sum_{i=1}^{\infty} u_{i}(\mathbf{x}, t) \psi_{i}(\boldsymbol{\xi}),
$$

where the coefficients $u_{i}(\mathbf{x}, t)$ are defined by the projections

$$
u_{i}(\mathbf{x}, t)=\left\langle u(\mathbf{x}, t, \boldsymbol{\xi}) \psi_{i}(\boldsymbol{\xi})\right\rangle, \quad i \in \mathbb{N} .
$$

The solution statistics are functions of the gPC coefficients and can easily be obtained by post-processing of the solution. For instance, the expectation and variance are given by, respectively,

$$
\mathbb{E}(u) \equiv\langle u\rangle=u_{1}, \quad \operatorname{Var}(u)=\sum_{i=2}^{\infty} u_{i}^{2},
$$

where the convention $\psi_{1}=1$ has been assumed. In practice, the infinite expansion in (1) is truncated to a finite number of terms such that, for instance, the total polynomial order of each $\psi_{i}(\boldsymbol{\xi})$ is at most $p$. The total number of gPC terms is then prescribed a priori, unless an adaptive method is used, in that case the number of basis functions change over time [17].

\section{Problem Formulation}

For ease of presentation, we consider the two-dimensional incompressible Navier-Stokes equations, while the proposed developments are applicable to the three-dimensional setting. Let $\mathbf{x}=(x, y)$ and consider the spatial domain $D=[0,2] \times[0,2]$. Assuming unit density, we have

$$
\begin{aligned}
u_{t}+u u_{x}+v u_{y}+p_{x}-\left[\left(\mu u_{x}\right)_{x}+\left(\mu u_{y}\right)_{y}\right] & =0 \\
v_{t}+u v_{x}+v v_{y}+p_{y}-\left[\left(\mu v_{x}\right)_{x}+\left(\mu v_{y}\right)_{y}\right] & =0 \\
u_{x}+v_{y} & =0 .
\end{aligned}
$$

In (4)-(6), $u$ and $v$ are the velocity components in the $x$ - and $y$-directions, respectively, $p$ is the pressure, and $\mu$ is the viscosity. The subscripts $x, y$ and $t$ refer to the spatial and temporal derivatives. Setting $\phi=u_{x}+v_{y}$ and taking the divergence of (4)-(5) without imposing (6), gives

$$
\phi_{t}+u \phi_{x}+v \phi_{y}-\left(\mu \phi_{x}\right)_{x}-\left(\mu \phi_{y}\right)_{y}=F,
$$


where

$$
F=\left(\mu_{x} u_{x}\right)_{x}+\left(\mu_{x} u_{y}\right)_{y}+\left(\mu_{y} v_{x}\right)_{x}+\left(\mu_{y} v_{y}\right)_{y}-u_{x}^{2}-2 u_{y} v_{x}-v_{y}^{2}-p_{x x}-p_{y y} .
$$

In the case of a constant $\mu$, which was considered in [13], we have $F=$ $-u_{x}^{2}-2 u_{y} v_{x}-v_{y}^{2}-p_{x x}-p_{y y}$.

The governing equations must be supplemented with suitable initial and boundary conditions. In this work we assume that all boundary data needed for a well-posed problem are available. The boundary conditions will be discussed in detail for the continuous case in Sections 4.1 and 4.2, and for the semi-discrete case in Sections 5.1 and 5.2.

In the numerical calculations we approximate the momentum equations (4)-(5) and the pressure equation (8) with $F=0$ within the order of accuracy of the numerical method chosen for the discretization. None of the equations are satisfied exactly on a finite computational grid, but we will derive a method that leads to high-order convergence in all solution variables $u, v, p$ and the divergence of the velocity converging to zero. Equation (7) is never solved explicitly through numerical discretization but instead is enforced implicitly through the enforcement of $F=0$ in (8) and suitable boundary conditions that lead to an energy estimate for the divergence. As a consequence, (6) is satisfied exactly in the continuous formulation and up to the order of accuracy of the numerical method in the discretized problem, without being directly imposed. This will be numerically demonstrated in Section 6.1.

\subsection{Stochastic Galerkin Formulation}

We next consider the case where the viscosity $\mu$ in (4)-(5) as well as the boundary and initial conditions are uncertain and characterized by an independent random vector $\boldsymbol{\xi}$. This implies that the velocity components $u, v$ and the pressure $p$ are also random functions, represented by the truncated 
gPC series

$$
\begin{aligned}
& u(\mathbf{x}, t, \boldsymbol{\xi})=\sum_{i=1}^{M} u_{i}(\mathbf{x}, t) \psi_{i}(\boldsymbol{\xi}) \\
& v(\mathbf{x}, t, \boldsymbol{\xi})=\sum_{i=1}^{M} v_{i}(\mathbf{x}, t) \psi_{i}(\boldsymbol{\xi}) \\
& p(\mathbf{x}, t, \boldsymbol{\xi})=\sum_{i=1}^{M} p_{i}(\mathbf{x}, t) \psi_{i}(\boldsymbol{\xi}) .
\end{aligned}
$$

The number of basis functions $M$ should be chosen sufficiently large to accurately represent the stochastic variation in the output, which could be unknown a priori. Spectral stochastic projection is performed by multiplying (4) and (5) with $\psi_{k}$ for $k=1, \ldots, M$ and integrating w.r.t. the measure $\mathcal{P}$. Following the stochastic Galerkin formulation, we require the residual of the equation to be orthogonal to each and every $\psi_{k}$, which leads to the following coupled systems of partial differential equations (PDEs) for the gPC expansion coefficients.

$$
\begin{aligned}
\left(u_{k}\right)_{t} & +\sum_{i=1}^{M} \sum_{j=1}^{M}\left[u_{i}\left(u_{j}\right)_{x}+v_{i}\left(u_{j}\right)_{y}\right]\left\langle\psi_{i} \psi_{j} \psi_{k}\right\rangle+\left(p_{k}\right)_{x} \\
& -\sum_{i=1}^{M^{\prime}} \sum_{j=1}^{M}\left[\left(\mu_{i}\left(u_{j}\right)_{x}\right)_{x}+\left(\mu_{i}\left(u_{j}\right)_{y}\right)_{y}\right]\left\langle\psi_{i} \psi_{j} \psi_{k}\right\rangle=0 \\
\left(v_{k}\right)_{t}+ & \sum_{i=1}^{M} \sum_{j=1}^{M}\left[u_{i}\left(v_{j}\right)_{x}+v_{i}\left(v_{j}\right)_{y}\right]\left\langle\psi_{i} \psi_{j} \psi_{k}\right\rangle+\left(p_{k}\right)_{y} \\
& -\sum_{i=1}^{M^{\prime}} \sum_{j=1}^{M}\left[\left(\mu_{i}\left(v_{j}\right)_{x}\right)_{x}+\left(\mu_{i}\left(v_{j}\right)_{y}\right)_{y}\right]\left\langle\psi_{i} \psi_{j} \psi_{k}\right\rangle=0 .
\end{aligned}
$$

The viscosity $\mu$ is represented by a gPC expansion of order $M^{\prime}$, where $M^{\prime} \geq$ $M$ is chosen sufficiently large for maximum accuracy of the projection of (4) and (5) on the finite space spanned by $\left\{\psi_{k}\right\}_{k=1}^{M}$.

Let $\mathbf{u}^{M}=\left(u_{1}, \ldots, u_{M}\right)^{T}$ and $\mathbf{v}^{M}=\left(v_{1}, \ldots, v_{M}\right)^{T}$ denote the vectors of gPC coefficients of $u$ and $v$, respectively. Let the matrix $\mathbf{A}\left(\mathbf{u}^{M}\right)$ be defined 
by

$$
\left[\mathbf{A}\left(\mathbf{u}^{M}\right)\right]_{k j}=\sum_{i=1}^{M} u_{i}\left\langle\psi_{i} \psi_{j} \psi_{k}\right\rangle, \quad j, k=1, \ldots, M .
$$

By the definition of $\mathbf{A}($.$) , for any vectors \mathbf{a}^{M}, \mathbf{b}^{M} \in \mathbb{R}^{M}$, it holds that

$$
\left[\mathbf{A}\left(\mathbf{a}^{M}\right) \mathbf{b}^{M}\right]_{k}=\sum_{j=1}^{M} \sum_{i=1}^{M}\left\langle\psi_{i} \psi_{j} \psi_{k}\right\rangle a_{i} b_{j}=\sum_{j=1}^{M} \sum_{i=1}^{M}\left\langle\psi_{i} \psi_{j} \psi_{k}\right\rangle b_{i} a_{j}=\left[\mathbf{A}\left(\mathbf{b}^{M}\right) \mathbf{a}^{M}\right]_{k}
$$

so we can interchange the vector valued argument of $\mathbf{A}($.$) with the vector$ the matrix is multiplied with, i.e. the order of the two factors of the product does not matter. Thus, we arrive at the expression

$$
\mathbf{A}\left(\mathbf{a}^{M}\right) \mathbf{b}^{M}=\mathbf{A}\left(\mathbf{b}^{M}\right) \mathbf{a}^{M},
$$

that is frequently used in our derivations. By using (14), the stochastic Galerkin formulation of the momentum equations (12)-(13) can be written

$$
\begin{aligned}
& \mathbf{u}_{t}^{M}+\mathbf{A}\left(\mathbf{u}^{M}\right) \mathbf{u}_{x}^{M}+\mathbf{A}\left(\mathbf{v}^{M}\right) \mathbf{u}_{y}^{M}+\mathbf{p}_{x}^{M}-\left(\left[\mathbf{A}(\boldsymbol{\mu}) \mathbf{u}_{x}^{M}\right]_{x}+\left[\mathbf{A}(\boldsymbol{\mu}) \mathbf{u}_{y}^{M}\right]_{y}\right)=\mathbf{0} \\
& \mathbf{v}_{t}^{M}+\mathbf{A}\left(\mathbf{u}^{M}\right) \mathbf{v}_{x}^{M}+\mathbf{A}\left(\mathbf{v}^{M}\right) \mathbf{v}_{y}^{M}+\mathbf{p}_{y}^{M}-\left(\left[\mathbf{A}(\boldsymbol{\mu}) \mathbf{v}_{x}^{M}\right]_{x}+\left[\mathbf{A}(\boldsymbol{\mu}) \mathbf{v}_{y}^{M}\right]_{y}\right)=\mathbf{0}
\end{aligned}
$$

where $\boldsymbol{\mu}=\left(\mu_{1}, \ldots, \mu_{M^{\prime}}\right)^{T}$ and $\mathbf{p}^{M}=\left(p_{1}, \ldots, p_{M}\right)^{T}$.

Remark 1. The order of the gPC expansion of $\mu$ is chosen sufficiently large to guarantee that $\mathbf{A}(\boldsymbol{\mu})$ is positive definite. This is explained and analyzed in [18] for a single stochastic dimension.

Performing stochastic Galerkin projection of the incompressibility condition (6) onto the stochastic basis yields

$$
\mathbf{u}_{x}^{M}+\mathbf{v}_{y}^{M}=\mathbf{0}
$$

Similarly, stochastic Galerkin projection of (7) gives

$$
\boldsymbol{\phi}_{t}^{M}+\mathbf{A}\left(\mathbf{u}^{M}\right) \boldsymbol{\phi}_{x}^{M}+\mathbf{A}\left(\mathbf{v}^{M}\right) \boldsymbol{\phi}_{y}^{M}-\left(\mathbf{A}(\boldsymbol{\mu}) \phi_{x}^{M}\right)_{x}-\left(\mathbf{A}(\boldsymbol{\mu}) \phi_{y}^{M}\right)_{y}=\mathbf{F}^{M}
$$

where

$$
\begin{array}{r}
\mathbf{F}^{M}=\left(\mathbf{A}\left(\boldsymbol{\mu}_{x}\right) \mathbf{u}_{x}^{M}\right)_{x}+\left(\mathbf{A}\left(\boldsymbol{\mu}_{x}\right) \mathbf{u}_{y}^{M}\right)_{y}+\left(\mathbf{A}\left(\boldsymbol{\mu}_{y}\right) \mathbf{v}_{x}^{M}\right)_{x}+\left(\mathbf{A}\left(\boldsymbol{\mu}_{y}\right) \mathbf{v}_{y}^{M}\right)_{y}-\mathbf{A}\left(\mathbf{u}_{x}^{M}\right) \mathbf{u}_{x}^{M} \\
-2 \mathbf{A}\left(\mathbf{u}_{y}^{M}\right) \mathbf{v}_{x}^{M}-\mathbf{A}\left(\mathbf{v}_{y}^{M}\right) \mathbf{v}_{y}^{M}-\mathbf{p}_{x x}^{M}-\mathbf{p}_{y y}^{M}
\end{array}
$$


Remark 2. The stochastic Galerkin version of the incompressibility condition is (17), i.e., a zero-divergence condition for each gPC coefficient of the velocity components.

Remark 3. Note that by setting $M=1$ in (15)-(19), we recover the deterministic case (4)-(8).

\section{Analysis of Well-posedness}

A well posed problem has a unique solution that depends continuously on input data. The latter property is here shown through an energy estimate. This is the main tool in the analysis of well-posedness, especially for problems with smooth solutions. It follows from the linearization and localization principles that if the constant coefficient and linearized formulation of an initial boundary value problem is well posed, then the corresponding nonlinear problem is also well posed $[19,20]$. In this section, we derive an energy estimate but defer the discussion on uniqueness and existence to future work.

As was indicated above, the incompressibility condition (17) is not explicitly enforced. As we will see, (17) is enforced indirectly by solving (18) with $\mathbf{F}^{M}=\mathbf{0}$ and a specific set of boundary conditions. To ensure incompressibility, we will derive an energy estimate that shows that incompressibility is indeed satisfied. The energy estimates in the following sections rely on time integration of expressions for the temporal derivative of the norm of the quantity of interest. Without subscripts specifying a weight function, $\|$. denotes the standard Euclidean norm.

\subsection{An Energy Estimate for Divergence Preservation}

We start by first left-multiplying the advection-diffusion equation (18) for the divergence gPC coefficients by $2\left(\phi^{M}\right)^{T}$. Next, we use that $\left(\boldsymbol{\phi}^{M}\right)^{T} \mathbf{A}\left(\mathbf{u}^{M}\right)=$ $\left(\mathbf{u}^{M}\right)^{T} \mathbf{A}\left(\boldsymbol{\phi}^{M}\right)$ and $\left(\boldsymbol{\phi}^{M}\right)^{T} \mathbf{A}\left(\mathbf{v}^{M}\right)=\left(\mathbf{v}^{M}\right)^{T} \mathbf{A}\left(\boldsymbol{\phi}^{M}\right)$ by (14). Finally, we integrate over the spatial domain to obtain the following expression for the time derivative of the norm of the velocity divergence.

$$
\begin{aligned}
& \frac{d}{d t}\left\|\boldsymbol{\phi}^{M}\right\|^{2}+\int_{D}\left(\mathbf{u}^{M}\right)^{T}\left[\mathbf{A}\left(\boldsymbol{\phi}^{M}\right) \boldsymbol{\phi}^{M}\right]_{x}+\left(\mathbf{v}^{M}\right)^{T}\left[\mathbf{A}\left(\boldsymbol{\phi}^{M}\right) \boldsymbol{\phi}^{M}\right]_{y} d x d y \\
& -2 \int_{D}\left(\boldsymbol{\phi}^{M}\right)^{T}\left[\mathbf{A}(\boldsymbol{\mu}) \boldsymbol{\phi}_{x}^{M}\right]_{x}+\left(\boldsymbol{\phi}^{M}\right)^{T}\left[\mathbf{A}(\boldsymbol{\mu}) \boldsymbol{\phi}_{y}^{M}\right]_{y} d x d y=2 \int_{D}\left(\boldsymbol{\phi}^{M}\right)^{T} \mathbf{F}^{M} d x d y
\end{aligned}
$$


Integration by parts and rearrangement of terms yield

$$
\begin{aligned}
\frac{d}{d t}\left\|\boldsymbol{\phi}^{M}\right\|^{2}+2 & \int_{D}\left(\boldsymbol{\phi}_{x}^{M}\right)^{T} \mathbf{A}(\boldsymbol{\mu}) \boldsymbol{\phi}_{x}^{M}+\left(\boldsymbol{\phi}_{y}^{M}\right)^{T} \mathbf{A}(\boldsymbol{\mu}) \boldsymbol{\phi}_{y}^{M} d x d y \\
& +\oint_{\partial D}\left(\mathbf{u}_{\mathbf{n}}^{M}\right)^{T} \mathbf{A}\left(\boldsymbol{\phi}^{M}\right) \boldsymbol{\phi}^{M}-2\left(\boldsymbol{\phi}^{M}\right)^{T} \mathbf{A}(\boldsymbol{\mu}) \frac{\partial \boldsymbol{\phi}^{M}}{\partial \mathbf{n}} d s \\
& =\int_{D}\left(\boldsymbol{\phi}^{M}\right)^{T} \mathbf{A}\left(\boldsymbol{\phi}^{M}\right) \boldsymbol{\phi}^{M}+2\left(\boldsymbol{\phi}^{M}\right)^{T} \mathbf{F}^{M} d x d y
\end{aligned}
$$

where $\mathbf{n}=\left(n_{1}, n_{2}\right)^{T}$ is the outward pointing normal, and we have adopted the notation

$$
\begin{aligned}
\mathbf{u}_{\mathbf{n}}^{M} & \equiv n_{1} \mathbf{u}^{M}+n_{2} \mathbf{v}^{M}, \\
\frac{\partial \boldsymbol{\phi}^{M}}{\partial \mathbf{n}} & \equiv n_{1} \boldsymbol{\phi}_{x}^{M}+n_{2} \boldsymbol{\phi}_{y}^{M} .
\end{aligned}
$$

In order to arrive at an energy estimate for the norm of the divergence coefficients, the boundary integral terms in (20) will be rewritten in a form that allows us to identify growth terms, which can then be controlled by subscription of boundary conditions. A systematic analysis to identify the most dissipative boundary conditions is presented in Apppendix A. Due to its relative simplicity of implementation, a practical choice of boundary conditions is

$$
\boldsymbol{\phi}^{M}=\mathbf{0}, \quad x, y \in \partial D .
$$

With these boundary conditions, the boundary integral terms in (20) vanish. For the volume terms on the right hand side of (20), we use the inequality

$$
2\left(\boldsymbol{\phi}^{M}\right)^{T} \mathbf{F}^{M} \leq \eta\left(\boldsymbol{\phi}^{M}\right)^{T} \boldsymbol{\phi}^{M}+\left(\mathbf{F}^{M}\right)^{T} \mathbf{F}^{M} / \eta, \quad \text { for any real } \eta>0 .
$$

Next we need to estimate the remaining cubic term on the right hand side of (20). Consider a limited time slot where $t \in\left[0, T_{0}\right]$ and let $\delta=\max _{t, x} \operatorname{eig}\left(\mathbf{A}\left(\boldsymbol{\phi}^{M}\right)\right)$. It holds that

$$
\int_{D}\left(\boldsymbol{\phi}^{M}\right)^{T} \mathbf{A}\left(\boldsymbol{\phi}^{M}\right) \boldsymbol{\phi}^{M}+2\left(\boldsymbol{\phi}^{M}\right)^{T} \mathbf{F}^{M} d x d y \leq(\delta+\eta)\left\|\boldsymbol{\phi}^{M}\right\|^{2}+\frac{1}{\eta}\left\|\mathbf{F}^{M}\right\|^{2} .
$$

Inserting the estimate (25) into (20), multiplying by the factor $e^{-(\delta+\eta) t}$ and 
integrating over time from 0 to $T_{0}$ leads to

$$
\begin{aligned}
\left\|\boldsymbol{\phi}^{M}\right\|^{2}+\int_{0}^{T_{0}} & {\left[2\left\|\boldsymbol{\phi}_{x}^{M}\right\|_{\mathbf{A}(\boldsymbol{\mu})}^{2}+2\left\|\boldsymbol{\phi}_{y}^{M}\right\|_{\mathbf{A}(\boldsymbol{\mu})}^{2}\right] e^{-(\delta+\eta)\left(t-T_{0}\right)} d t } \\
& \leq\left\|\boldsymbol{\phi}^{M}(t=0)\right\|^{2} e^{(\delta+\eta) T_{0}}+\frac{1}{\eta} \int_{0}^{T_{0}}\left\|\mathbf{F}^{M}\right\|^{2} e^{-(\delta+\eta)\left(t-T_{0}\right)} d t .
\end{aligned}
$$

We impose zero initial data, $\boldsymbol{\phi}^{M}(t=0)=\mathbf{0}$, and require that $\mathbf{F}^{M}=\mathbf{0}$. Then (26) implies that $\phi^{M}\left(t \leq T_{0}\right) \equiv 0$. Using $\phi^{M}\left(t=T_{0}\right)=0$ as an initial condition, the argument is repeated for the time slot $\left[T_{0}, 2 T_{0}\right]$. Note that if the argument holds for some choice of $T_{0}$, it must hold for any choice of $T_{0}$. Successively repeating the argument, each time starting with the initial condition $\phi^{M} \equiv 0$ from the previous step, it follows by induction that the zero divergence condition is satisfied for all times.

\subsection{An Energy Estimate for the Momentum Equations}

The derivation of an energy estimate for the stochastic Galerkin momentum equations is a generalization of the deterministic case. For completeness and appreciation of the similarities and differences imposed by the stochastic Galerkin formulation, in Section 4.2.1 we start with the deterministic derivation published in [13]. With a roadmap given by that analysis, we then proceed with the analysis of the stochastic Galerkin formulation of the momentum equations in Section 4.2.2.

\subsubsection{The Deterministic Case}

Multiplying the momentum equations (4)-(5) from the left with the velocities and integrating over the spatial domain $D$, we get

$$
\begin{aligned}
& \frac{d}{d t}\left(\|u\|^{2}+\|v\|^{2}\right)+2 \int_{D} \underbrace{u^{2} u_{x}+u v u_{y}+u v v_{x}+v^{2} v_{y}}_{\frac{1}{2} u\left(u^{2}+v^{2}\right)_{x}+\frac{1}{2} v\left(u^{2}+v^{2}\right)_{y}} d x d y \\
+ & 2 \int_{D}\left(u p_{x}+v p_{y}\right) d x d y-2 \int_{D}\left(u\left(\mu u_{x}\right)_{x}+u\left(\mu u_{y}\right)_{y}+v\left(\mu v_{x}\right)_{x}+v\left(\mu v_{y}\right)_{y}\right) d x d y=0 .
\end{aligned}
$$


Integration by parts of (27) yields

$$
\begin{aligned}
& \frac{d}{d t}\left(\|u\|^{2}+\|v\|^{2}\right)+\underbrace{\oint_{\partial D}\left(u^{2}+v^{2}\right)\left(u n_{1}+v n_{2}\right) d s-\int_{D}\left(u_{x}+v_{y}\right)\left(u^{2}+v^{2}\right) d x d y} \\
& \text { Advective terms } \\
& +\underbrace{2 \oint_{\partial D}\left(u n_{1}+v n_{2}\right) p d s-2 \int_{D}\left(u_{x}+v_{y}\right) p d x d y} \\
& \text { Pressure terms } \\
& \underbrace{-2 \oint_{\partial D} \mu\left(u u_{x}+u u_{y}+v v_{x}+v v_{y}\right) d s+2\left(\|\nabla u\|_{\mu}^{2}+\|\nabla v\|_{\mu}^{2}\right)}=0,
\end{aligned}
$$

\section{Viscous terms}

where $\mathbf{n}=\left(n_{1}, n_{2}\right)^{T}$ is the outward pointing normal to $\partial D$. It was shown in Section 4.1 that $\boldsymbol{\phi}^{M}=\mathbf{u}_{x}^{M}+\mathbf{v}_{y}^{M}=\mathbf{0}$ for all $M$ including $M=1$ which is equivalent to the deterministic case. Thus, the volume integrals related to the advective and pressure terms in (28) vanish. The boundary integral terms can be rewritten as

$$
\begin{array}{r}
\oint_{\partial D}\left(u^{2}+v^{2}\right)\left(u n_{1}+v n_{2}\right)+2\left(u n_{1}+v n_{2}\right) p-2 \mu\left(u u_{x}+u u_{y}+v v_{x}+v v_{y}\right) d s \\
=\oint_{\partial D} \mathbf{w}^{T} \mathbf{B} \mathbf{w} d s
\end{array}
$$

where

$$
\mathbf{w}=\left(\begin{array}{c}
u_{\mathbf{n}} \\
u_{\mathbf{s}} \\
p \\
\mu \frac{\partial u_{\mathbf{n}}}{\partial \mathbf{n}} \\
\mu \frac{\partial u_{\mathbf{s}}}{\partial \mathbf{n}}
\end{array}\right), \quad \mathbf{B}=\left(\begin{array}{ccccc}
u_{\mathbf{n}} & 0 & 1 & -1 & 0 \\
0 & u_{\mathbf{n}} & 0 & 0 & -1 \\
1 & 0 & 0 & 0 & 0 \\
-1 & 0 & 0 & 0 & 0 \\
0 & -1 & 0 & 0 & 0
\end{array}\right)
$$

The eigenvalues of $\mathbf{B}$ are

$$
\lambda_{1,2}=\frac{u_{\mathbf{n}}}{2} \pm \sqrt{\left(\frac{u_{\mathbf{n}}}{2}\right)^{2}+1}, \quad \lambda_{3}=0, \quad \lambda_{4,5}=\frac{u_{\mathbf{n}}}{2} \pm \sqrt{\left(\frac{u_{\mathbf{n}}}{2}\right)^{2}+1} .
$$

We impose the boundary conditions $\left(\mathbf{X}^{T} \mathbf{w}\right)_{j}=g_{j}$ for $j=2,5$ (corresponding to the negative eigenvalues $\lambda_{2}$ and $\lambda_{5}$ ) with $\mathbf{X}$ being the matrix of eigenvectors of $\mathbf{B}$. This ensures that the correct number of boundary conditions is imposed. 


\subsubsection{The Stochastic Galerkin Case}

Multiply (15)-(16) by $\left[\left(\mathbf{u}^{M}\right)^{T},\left(\mathbf{v}^{M}\right)^{T}\right]$ and integrate over the spatial domain. The resulting expression can be written

$\frac{d}{d t}\left(\left\|\mathbf{u}^{M}\right\|^{2}+\left\|\mathbf{v}^{M}\right\|^{2}\right)+$ advective terms + pressure terms + diffusive terms $=0$.

For clarity, we define and treat the terms groupwise below.

For the advective terms, we use that $\mathbf{A}($.$) is linear in its argument to$ rewrite the spatial derivatives. Then we perform integration by parts,

$$
\begin{gathered}
2 \int_{D}\left(\left(\mathbf{u}^{M}\right)^{T} \mathbf{A}\left(\mathbf{u}^{M}\right) \mathbf{u}_{x}^{M}+\left(\mathbf{v}^{M}\right)^{T} \mathbf{A}\left(\mathbf{u}^{M}\right) \mathbf{v}_{x}^{M}+\left(\mathbf{u}^{M}\right)^{T} \mathbf{A}\left(\mathbf{v}^{M}\right) \mathbf{u}_{y}^{M}\right) d x d y \\
+2 \int_{D}\left(\mathbf{v}^{M}\right)^{T} \mathbf{A}\left(\mathbf{v}^{M}\right) \mathbf{v}_{y}^{M} d x d y \\
=\int_{D}\left(\left(\mathbf{u}^{M}\right)^{T}\left[\mathbf{A}\left(\mathbf{u}^{M}\right) \mathbf{u}^{M}+\mathbf{A}\left(\mathbf{v}^{M}\right) \mathbf{v}^{M}\right]_{x}+\left(\mathbf{v}^{M}\right)^{T}\left[\mathbf{A}\left(\mathbf{u}^{M}\right) \mathbf{u}^{M}+\mathbf{A}\left(\mathbf{v}^{M}\right) \mathbf{v}^{M}\right]_{y}\right) d x d y \\
=\oint_{\partial D}\left(\mathbf{u}^{M} n_{1}+\mathbf{v}^{M} n_{2}\right)^{T}\left[\mathbf{A}\left(\mathbf{u}^{M}\right) \mathbf{u}^{M}+\mathbf{A}\left(\mathbf{v}^{M}\right) \mathbf{v}^{M}\right] d s \\
-\int_{D}\left(\phi^{M}\right)^{T}\left[\mathbf{A}\left(\mathbf{u}^{M}\right) \mathbf{u}^{M}+\mathbf{A}\left(\mathbf{v}^{M}\right) \mathbf{v}^{M}\right] d x d y .
\end{gathered}
$$

In the first equality, we used that $2 \mathbf{A}\left(\mathbf{u}^{M}\right) \mathbf{u}_{x}^{M}=\mathbf{A}\left(\mathbf{u}^{M}\right) \mathbf{u}_{x}^{M}+\mathbf{A}\left(\mathbf{u}_{x}^{M}\right) \mathbf{u}^{M}=$ $\left[\mathbf{A}\left(\mathbf{u}^{M}\right) \mathbf{u}^{M}\right]_{x}$, and similar expressions for $\mathbf{v}^{M}$ and the $y$-derivatives.

For the pressure terms, we perform integration by parts,

$2 \int_{D}\left(\mathbf{u}^{M}\right)^{T} \mathbf{p}_{x}^{M}+\left(\mathbf{v}^{M}\right)^{T} \mathbf{p}_{y}^{M} d x d y=2 \oint_{\partial D}\left(\mathbf{u}^{M} n_{1}+\mathbf{v}^{M} n_{2}\right)^{T} \mathbf{p}^{M} d s-2 \int_{D}\left(\phi^{M}\right)^{T} \mathbf{p}^{M} d x d y$.

For the diffusive terms, we perform integration by parts and rewrite the resulting volume integral, assuming that $\mathbf{A}(\boldsymbol{\mu})$ defines a norm, i.e. that $\mathbf{A}(\boldsymbol{\mu})$ is a positive definite matrix. This is a valid assumption since $\mu$ is 
positive almost surely (in the probabilistic sense). We obtain

$$
\begin{aligned}
& 2 \int_{D}\left(\left(\mathbf{u}^{M}\right)^{T}\left[\mathbf{A}(\boldsymbol{\mu}) \mathbf{u}_{x}^{M}\right]_{x}+\left(\mathbf{u}^{M}\right)^{T}\left[\mathbf{A}(\boldsymbol{\mu}) \mathbf{u}_{y}^{M}\right]_{y}+\left(\mathbf{v}^{M}\right)^{T}\left[\mathbf{A}(\boldsymbol{\mu}) \mathbf{v}_{x}^{M}\right]_{x}\right) d x d y \\
& +2 \int_{D}\left(\mathbf{v}^{M}\right)^{T}\left[\mathbf{A}(\boldsymbol{\mu}) \mathbf{v}_{y}^{M}\right]_{y} d x d y=2 \oint_{\partial D} n_{1}\left(\mathbf{u}^{M}\right)^{T} \mathbf{A}(\boldsymbol{\mu}) \mathbf{u}_{x}^{M}+n_{2}\left(\mathbf{u}^{M}\right)^{T} \mathbf{A}(\boldsymbol{\mu}) \mathbf{u}_{y}^{M} d s \\
& \quad+2 \oint_{\partial D} n_{1}\left(\mathbf{v}^{M}\right)^{T} \mathbf{A}(\boldsymbol{\mu}) \mathbf{v}_{x}^{M}+n_{2}\left(\mathbf{v}^{M}\right)^{T} \mathbf{A}(\boldsymbol{\mu}) \mathbf{v}_{y}^{M} d s \\
& -2 \underbrace{\int_{D}\left(\left(\mathbf{u}_{x}^{M}\right)^{T} \mathbf{A}(\boldsymbol{\mu}) \mathbf{u}_{x}^{M}+\left(\mathbf{u}_{y}^{M}\right)^{T} \mathbf{A}(\boldsymbol{\mu}) \mathbf{u}_{y}^{M}+\left(\mathbf{v}_{x}^{M}\right)^{T} \mathbf{A}(\boldsymbol{\mu}) \mathbf{v}_{x}^{M}+\left(\mathbf{v}_{y}^{M}\right)^{T} \mathbf{A}(\boldsymbol{\mu}) \mathbf{v}_{y}^{M}\right) d x d y}_{\left\|\mathbf{u}_{x}^{M}\right\|_{\mathbf{A}(\boldsymbol{\mu})}^{2}+\left\|\mathbf{v}_{x}^{M}\right\|_{\mathbf{A}(\boldsymbol{\mu})}^{2}+\left\|\mathbf{u}_{y}^{M}\right\|_{\mathbf{A}(\boldsymbol{\mu})}^{2}+\left\|\mathbf{v}_{y}^{M}\right\|_{\mathbf{A}(\boldsymbol{\mu})}^{2}} .
\end{aligned}
$$

Assembling the advective, pressure and diffusive terms, the expression (31) becomes

$$
\begin{aligned}
& \frac{d}{d t}\left(\left\|\mathbf{u}^{M}\right\|^{2}+\left\|\mathbf{v}^{M}\right\|^{2}\right)+2\left[\left\|\mathbf{u}_{x}^{M}\right\|_{\mathbf{A}(\boldsymbol{\mu})}^{2}+\left\|\mathbf{v}_{x}^{M}\right\|_{\mathbf{A}(\boldsymbol{\mu})}^{2}+\left\|\mathbf{u}_{y}^{M}\right\|_{\mathbf{A}(\boldsymbol{\mu})}^{2}+\left\|\mathbf{v}_{y}^{M}\right\|_{\mathbf{A}(\boldsymbol{\mu})}^{2}\right] \\
& =-2 \oint_{\partial D}\left(\mathbf{u}^{M} n_{1}+\mathbf{v}^{M} n_{2}\right)^{T}\left[\mathbf{A}\left(\mathbf{u}^{M}\right) \mathbf{u}^{M} / 2+\mathbf{A}\left(\mathbf{v}^{M}\right) \mathbf{v}^{M} / 2+\mathbf{p}^{M}\right] d s \\
& +2 \oint_{\partial D} n_{1}\left(\mathbf{u}^{M}\right)^{T} \mathbf{A}(\boldsymbol{\mu}) \mathbf{u}_{x}^{M}+n_{2}\left(\mathbf{u}^{M}\right)^{T} \mathbf{A}(\boldsymbol{\mu}) \mathbf{u}_{y}^{M}+n_{1}\left(\mathbf{v}^{M}\right)^{T} \mathbf{A}(\boldsymbol{\mu}) \mathbf{v}_{x}^{M} d s \\
& +2 \oint_{\partial D} n_{2}\left(\mathbf{v}^{M}\right)^{T} \mathbf{A}(\boldsymbol{\mu}) \mathbf{v}_{y}^{M} d s+2 \int_{D}\left(\boldsymbol{\phi}^{M}\right)^{T}\left[\mathbf{A}\left(\mathbf{u}^{M}\right) \mathbf{u}^{M} / 2+\mathbf{A}\left(\mathbf{v}^{M}\right) \mathbf{v}^{M} / 2+\mathbf{p}^{M}\right] d x d y .
\end{aligned}
$$

In order to rewrite (32) to obtain an energy estimate, we use $\mathbf{s}=\left(n_{2},-n_{1}\right)^{T}$ to denote the vector tangential to the spatial boundary $\partial D$. Let

$$
\begin{aligned}
& \mathbf{u}_{\mathbf{s}}^{M} \equiv n_{2} \mathbf{u}^{M}-n_{1} \mathbf{v}^{M}, \\
& \frac{\partial \mathbf{u}_{\mathbf{n}}^{M}}{\partial \mathbf{n}} \equiv n_{1}\left(\mathbf{u}_{n}^{M}\right)_{x}+n_{2}\left(\mathbf{u}_{n}^{M}\right)_{y}, \\
& \frac{\partial \mathbf{u}_{\mathbf{s}}^{M}}{\partial \mathbf{n}} \equiv n_{1}\left(\mathbf{u}_{s}^{M}\right)_{x}+n_{2}\left(\mathbf{u}_{s}^{M}\right)_{y} .
\end{aligned}
$$

By combining the property (14) of matrix $\mathbf{A}($.$) with (21) and (33), we get$ the identity

$$
\begin{aligned}
& \mathbf{A}\left(\mathbf{u}_{\mathbf{n}}^{M}\right) \mathbf{u}_{\mathbf{n}}^{M}+\mathbf{A}\left(\mathbf{u}_{\mathbf{s}}^{M}\right) \mathbf{u}_{\mathbf{s}}^{M}=\left[n_{1} \mathbf{A}\left(\mathbf{u}^{M}\right)+n_{2} \mathbf{A}\left(\mathbf{v}^{M}\right)\right]\left[n_{1} \mathbf{u}^{M}+n_{2} \mathbf{v}^{M}\right] \\
&+\left[n_{2} \mathbf{A}\left(\mathbf{u}^{M}\right)-\right.\left.n_{1} \mathbf{A}\left(\mathbf{v}^{M}\right)\right]\left[n_{2} \mathbf{u}^{M}-n_{1} \mathbf{v}^{M}\right]=\left(n_{1}^{2}+n_{2}^{2}\right) \mathbf{A}\left(\mathbf{u}^{M}\right) \mathbf{u}^{M} \\
&+\left(n_{1}^{2}+n_{2}^{2}\right) \mathbf{A}\left(\mathbf{v}^{M}\right) \mathbf{v}^{M}=\mathbf{A}\left(\mathbf{u}^{M}\right) \mathbf{u}^{M}+\mathbf{A}\left(\mathbf{v}^{M}\right) \mathbf{v}^{M} .
\end{aligned}
$$


Also, using (21) and (33)-(35), we have

$$
\begin{array}{rl}
\left(\mathbf{u}_{\mathbf{n}}^{M}\right)^{T} & \mathbf{A}(\boldsymbol{\mu}) \frac{\partial \mathbf{u}_{\mathbf{n}}^{M}}{\partial \mathbf{n}}+\left(\mathbf{u}_{\mathbf{s}}^{M}\right)^{T} \mathbf{A}(\boldsymbol{\mu}) \frac{\partial \mathbf{u}_{\mathbf{s}}^{M}}{\partial \mathbf{n}} \\
= & \left(n_{1} \mathbf{u}^{M}+n_{2} \mathbf{v}^{M}\right)^{T} \mathbf{A}(\boldsymbol{\mu})\left[n_{1}^{2} \mathbf{u}_{x}^{M}+n_{1} n_{2} \mathbf{v}_{x}^{M}+n_{1} n_{2} \mathbf{u}_{y}^{M}+n_{2}^{2} \mathbf{v}_{y}^{M}\right] \\
+ & \left(n_{2} \mathbf{u}^{M}-n_{1} \mathbf{v}^{M}\right)^{T} \mathbf{A}(\boldsymbol{\mu})\left[n_{1} n_{2} \mathbf{u}_{x}^{M}-n_{1}^{2} \mathbf{v}_{x}^{M}+n_{2}^{2} \mathbf{u}_{y}^{M}-n_{1} n_{2} \mathbf{v}_{y}^{M}\right] \\
= & n_{1}\left(n_{1}^{2}+n_{2}^{2}\right)\left(\mathbf{u}^{M}\right)^{T} \mathbf{A}(\boldsymbol{\mu}) \mathbf{u}_{x}^{M}+n_{2}\left(n_{1}^{2}+n_{2}^{2}\right)\left(\mathbf{u}^{M}\right)^{T} \mathbf{A}(\boldsymbol{\mu}) \mathbf{u}_{y}^{M} \\
& +n_{1}\left(n_{1}^{2}+n_{2}^{2}\right)\left(\mathbf{v}^{M}\right)^{T} \mathbf{A}(\boldsymbol{\mu}) \mathbf{v}_{x}^{M}+n_{2}\left(n_{1}^{2}+n_{2}^{2}\right)\left(\mathbf{v}^{M}\right)^{T} \mathbf{A}(\boldsymbol{\mu})_{y}^{M} \\
=n_{1}\left(\mathbf{u}^{M}\right)^{T} \mathbf{A}(\boldsymbol{\mu}) \mathbf{u}_{x}^{M}+n_{2}\left(\mathbf{u}^{M}\right)^{T} \mathbf{A}(\boldsymbol{\mu}) \mathbf{u}_{y}^{M}+n_{1}\left(\mathbf{v}^{M}\right)^{T} \mathbf{A}(\boldsymbol{\mu}) \mathbf{v}_{x}^{M}+n_{2}\left(\mathbf{v}^{M}\right)^{T} \mathbf{A}(\boldsymbol{\mu}) \mathbf{v}_{y}^{M} .
\end{array}
$$

Finally, by combining (36), (37) and (32), we obtain,

$$
\begin{aligned}
& \frac{d}{d t}\left(\left\|\mathbf{u}^{M}\right\|^{2}+\left\|\mathbf{v}^{M}\right\|^{2}\right)+2\left[\left\|\mathbf{u}_{x}^{M}\right\|_{\mathbf{A}(\boldsymbol{\mu})}^{2}+\left\|\mathbf{v}_{x}^{M}\right\|_{\mathbf{A}(\boldsymbol{\mu})}^{2}+\left\|\mathbf{u}_{y}^{M}\right\|_{\mathbf{A}(\boldsymbol{\mu})}^{2}+\left\|\mathbf{v}_{y}^{M}\right\|_{\mathbf{A}(\boldsymbol{\mu})}^{2}\right] \\
= & -\oint_{\partial D}\left(\mathbf{w}^{M}\right)^{T} \mathbf{B}^{M} \mathbf{w}^{M} d s+2 \int_{D}\left(\boldsymbol{\phi}^{M}\right)^{T}\left[\mathbf{A}\left(\mathbf{u}^{M}\right) \mathbf{u}^{M} / 2+\mathbf{A}\left(\mathbf{v}^{M}\right) \mathbf{v}^{M} / 2+\mathbf{p}^{M}\right] d x d y
\end{aligned}
$$

where

$$
\mathbf{W}^{M}=\left[\begin{array}{c}
\mathbf{u}_{\mathbf{n}}^{M} \\
\mathbf{u}_{\mathbf{s}}^{M} \\
\mathbf{p}^{M} \\
\mathbf{A}(\boldsymbol{\mu}) \frac{\partial \mathbf{u}_{\mathbf{n}}^{M}}{\partial \mathbf{n}^{M}} \\
\mathbf{A}(\boldsymbol{\mu}) \frac{\partial \mathbf{u}_{\mathbf{s}}}{\partial \mathbf{n}}
\end{array}\right], \quad \mathbf{B}^{M}=\left[\begin{array}{ccccc}
\mathbf{A}\left(\mathbf{u}_{\mathbf{n}}^{M}\right) & \mathbf{0} & \mathbf{I}^{M} & -\mathbf{I}^{M} & \mathbf{0} \\
\mathbf{0} & \mathbf{A}\left(\mathbf{u}_{\mathbf{n}}^{M}\right) & \mathbf{0} & \mathbf{0} & -\mathbf{I}^{M} \\
\mathbf{I}^{M} & \mathbf{0} & \mathbf{0} & \mathbf{0} & \mathbf{0} \\
-\mathbf{I}^{M} & \mathbf{0} & \mathbf{0} & \mathbf{0} & \mathbf{0} \\
\mathbf{0} & -\mathbf{I}^{M} & \mathbf{0} & \mathbf{0} & \mathbf{0}
\end{array}\right]
$$

Note that (39) mimics the expression (13) in [13] and (29) in this paper. Just as in the deterministic case, we need the sign of the eigenvalues of $\mathbf{B}^{M}$ to be able to impose boundary conditions.

\subsubsection{Eigenvalues of $\mathbf{B}^{M}$ and Imposition of Boundary Conditions}

To find the eigenvalues of $\mathbf{B}^{M}$, consider the equivalent problem of finding the eigenvalues of a similar matrix. Let $\mathbf{X}_{\mathbf{u}_{\mathbf{n}}}$ be the matrix of eigenvectors and $\Lambda_{\mathbf{u}_{\mathbf{n}}}$ be the diagonal matrix of eigenvalues of the eigenvalue decomposition 
$\mathbf{A}\left(\mathbf{u}_{\mathbf{n}}^{M}\right)=\mathbf{X}_{\mathbf{u}_{\mathbf{n}}} \boldsymbol{\Lambda}_{\mathbf{u}_{\mathbf{n}}} \mathbf{X}_{\mathbf{u}_{\mathbf{n}}}^{T}$. Then, the matrix $\mathbf{B}_{\boldsymbol{\Lambda}}^{M}$, defined by

$$
\mathbf{B}_{\Lambda}^{M}=\left[\begin{array}{ccccc}
\Lambda_{\mathbf{u}_{\mathbf{n}}} & \mathbf{0} & \mathbf{I}^{M} & -\mathbf{I}^{M} & \mathbf{0} \\
\mathbf{0} & \boldsymbol{\Lambda}_{\mathbf{u}_{\mathbf{n}}} & \mathbf{0} & \mathbf{0} & -\mathbf{I}^{M} \\
\mathbf{I}^{M} & \mathbf{0} & \mathbf{0} & \mathbf{0} & \mathbf{0} \\
-\mathbf{I}^{M} & \mathbf{0} & \mathbf{0} & \mathbf{0} & \mathbf{0} \\
\mathbf{0} & -\mathbf{I}^{M} & \mathbf{0} & \mathbf{0} & \mathbf{0}
\end{array}\right]=\left(\mathbf{I}^{5} \otimes \mathbf{X}_{\mathbf{u}_{\mathbf{n}}}^{T}\right) \mathbf{B}^{M}\left(\mathbf{I}^{5} \otimes \mathbf{X}_{\mathbf{u}_{\mathbf{n}}}\right)
$$

is similar to $\mathbf{B}^{M}$. Thus,

$$
0=\operatorname{det}\left(\lambda \mathbf{I}^{5 M}-\mathbf{B}^{M}\right)=\operatorname{det}\left[\begin{array}{cc|ccc}
\lambda \mathbf{I}^{M}-\boldsymbol{\Lambda}_{\mathbf{u}_{\mathbf{n}}} & \mathbf{0} & -\mathbf{I}^{M} & \mathbf{I}^{M} & \mathbf{0} \\
\mathbf{0} & \lambda \mathbf{I}^{M}-\boldsymbol{\Lambda}_{\mathbf{u}_{\mathbf{n}}} & \mathbf{0} & \mathbf{0} & \mathbf{I}^{M} \\
\hline-\mathbf{I}^{M} & \mathbf{0} & \lambda \mathbf{I}^{M} & \mathbf{0} & \mathbf{0} \\
\mathbf{I}^{M} & \mathbf{0} & \mathbf{0} & \lambda \mathbf{I}^{M} & \mathbf{0} \\
\mathbf{0} & \mathbf{I}^{M} & \mathbf{0} & \mathbf{0} & \lambda \mathbf{I}^{M}
\end{array}\right]
$$

where $\mathbf{I}^{5 M}$ is the identity matrix of size $5 M \times 5 M$. We will use the fact that for any matrices $\hat{\mathbf{A}} \in \mathbb{R}^{m \times m}, \hat{\mathbf{B}} \in \mathbb{R}^{m \times n}, \hat{\mathbf{C}} \in \mathbb{R}^{n \times m}$ and $\hat{\mathbf{D}} \in \mathbb{R}^{n \times n}$ it holds that

$$
\begin{array}{r}
\operatorname{det}\left[\begin{array}{cc}
\hat{\mathbf{A}} & \hat{\mathbf{B}} \\
\hat{\mathbf{C}} & \hat{\mathbf{D}}
\end{array}\right]=\operatorname{det}\left(\left[\begin{array}{ll}
\mathbf{I} & \hat{\mathbf{B}} \\
\mathbf{0} & \hat{\mathbf{D}}
\end{array}\right]\left[\begin{array}{cc}
\hat{\mathbf{A}}-\hat{\mathbf{B}}^{-1} \hat{\mathbf{C}} & \mathbf{0} \\
\hat{\mathbf{D}}^{-1} \hat{\mathbf{C}} & \mathbf{I}
\end{array}\right]\right) \\
=\operatorname{det}(\hat{\mathbf{D}}) \operatorname{det}\left(\hat{\mathbf{A}}-\hat{\mathbf{B}}^{-1} \hat{\mathbf{C}}\right),
\end{array}
$$

assuming that $\hat{\mathbf{D}}$ is invertible [21]. Let

$$
\hat{\mathbf{A}}=\left(\lambda \mathbf{I}^{M}-\boldsymbol{\Lambda}_{\mathbf{u}_{\mathbf{n}}}\right) \otimes \mathbf{I}^{2}, \quad \hat{\mathbf{B}}=\hat{\mathbf{C}}^{T}=\left[\begin{array}{ccc}
-\mathbf{I}^{M} & \mathbf{I}^{M} & \mathbf{0} \\
\mathbf{0} & \mathbf{0} & \mathbf{I}^{M}
\end{array}\right], \quad \hat{\mathbf{D}}=\lambda \mathbf{I}^{3 M}
$$

Then, by (40)

$$
\begin{gathered}
0=\operatorname{det}\left(\lambda \mathbf{I}^{5 M}-\mathbf{B}^{M}\right)= \\
=\operatorname{det}\left[\begin{array}{ccc}
\lambda \mathbf{I}^{M} & \mathbf{0} & \mathbf{0} \\
\mathbf{0} & \lambda \mathbf{I}^{M} & \mathbf{0} \\
\mathbf{0} & \mathbf{0} & \lambda \mathbf{I}^{M}
\end{array}\right] \operatorname{det}\left[\begin{array}{cc}
\lambda \mathbf{I}^{M}-\boldsymbol{\Lambda}_{\mathbf{u}_{\mathbf{n}}}-2 \lambda^{-1} \mathbf{I}^{M} & \mathbf{0} \\
\mathbf{0} & \lambda \mathbf{I}^{M}-\boldsymbol{\Lambda}_{\mathbf{u}_{\mathbf{n}}}-\lambda^{-1} \mathbf{I}^{M}
\end{array}\right] \\
=\lambda^{3 M} \operatorname{det}\left(\operatorname{diag}\left(\lambda \mathbf{I}^{M}-\boldsymbol{\Lambda}_{\mathbf{u}_{\mathbf{n}}}-2 \lambda^{-1} \mathbf{I}^{M}, \lambda \mathbf{I}^{M}-\boldsymbol{\Lambda}_{\mathbf{u}_{\mathbf{n}}}-\lambda^{-1} \mathbf{I}^{M}\right)\right) \\
=\lambda^{M} \operatorname{det}\left(\operatorname{diag}\left(\lambda^{2} \mathbf{I}^{M}-\lambda \boldsymbol{\Lambda}_{\mathbf{u}_{\mathbf{n}}}-2 \mathbf{I}^{M}, \lambda^{2} \mathbf{I}^{M}-\lambda \boldsymbol{\Lambda}_{\mathbf{u}_{\mathbf{n}}}-\mathbf{I}^{M}\right)\right)
\end{gathered}
$$


The superscripts on $\lambda$ denote powers, but superscripts on matrices (boldface) always denote the order of gPC. The solutions $\lambda$ of (41) are

$$
\begin{aligned}
& \lambda_{1, j}=\frac{\lambda_{\mathbf{u}_{\mathbf{n}}, j}}{2}+\sqrt{\frac{\lambda_{\mathbf{u}_{\mathbf{n}}, j}^{2}}{4}+1}, \quad j=1, \ldots, M, \\
& \lambda_{2, j}=\frac{\lambda_{\mathbf{u}_{\mathbf{n}}, j}}{2}-\sqrt{\frac{\lambda_{\mathbf{u}_{\mathbf{n}}, j}^{2}}{4}+1}, \quad j=1, \ldots, M, \\
& \lambda_{3, j}=0, \quad \text { multiplicity } M, \quad j=1, \ldots, M \text {, } \\
& \lambda_{4, j}=\frac{\lambda_{\mathbf{u}_{\mathbf{n}}, j}}{2}+\sqrt{\frac{\lambda_{\mathbf{u}_{\mathbf{n}, j}}^{2}}{4}+2}, \quad j=1, \ldots, M, \\
& \lambda_{5, j}=\frac{\lambda_{\mathbf{u}_{\mathbf{n}}, j}}{2}-\sqrt{\frac{\lambda_{\mathbf{u}_{\mathbf{n}}, j}^{2}}{4}+2}, \quad j=1, \ldots, M .
\end{aligned}
$$

The subscript $\mathbf{u}_{\mathbf{n}}$ refers to the velocity in the normal direction $\mathbf{n}$. As was mentioned above, the stochastic Galerkin eigenvalues (42)-(46) are direct generalizations of the deterministic eigenvalues (30).

Similarly to the deterministic case, the negative eigenvalues are always $\lambda_{2, j}$ and $\lambda_{5, j}, j=1 \ldots, M$, independent of both the mean flow direction and the sign of higher order coefficients. The number of boundary conditions therefore remains exactly $2 M$.

Remark 4. The fixed number of boundary conditions will be a great advantage for the numerical solution of the problem since in this case no special considerations are needed for characteristic curves changing direction. This situation is far from self-evident, and this is in fact not always the case even for scalar linear problems. For example, c.f. [22] for a stochastic Galerkin formulation of an advection equation where the number of boundary conditions depends on the input uncertainty.

The boundary conditions are prescribed on the characteristics corresponding to the negative eigenvalues. We have

$$
\begin{array}{r}
\mathbf{X}_{\mathbf{u}_{\mathbf{n}}} \boldsymbol{\Lambda}_{2} \mathbf{X}_{\mathbf{u}_{\mathbf{n}}}^{T} \mathbf{u}_{\mathbf{s}}^{M}-\mathbf{A}(\boldsymbol{\mu}) \frac{\partial \mathbf{u}_{\mathbf{s}}^{M}}{\partial \mathbf{n}}=\mathbf{g}_{2}^{M}, \\
\mathbf{X}_{\mathbf{u}_{\mathbf{n}}} \boldsymbol{\Lambda}_{5} \mathbf{X}_{\mathbf{u}_{\mathbf{n}}}^{T} \mathbf{u}_{\mathbf{n}}^{M}+\mathbf{p}^{M}-\mathbf{A}(\boldsymbol{\mu}) \frac{\partial \mathbf{u}_{\mathbf{n}}^{M}}{\partial \mathbf{n}}=\mathbf{g}_{5}^{M},
\end{array}
$$


where $\boldsymbol{\Lambda}_{2}=\operatorname{diag}\left(\lambda_{2,1}, \ldots, \lambda_{2, M}\right)$ and $\boldsymbol{\Lambda}_{5}=\operatorname{diag}\left(\lambda_{5,1}, \ldots, \lambda_{5, M}\right)$.

We showed in Section 4.1 that $\boldsymbol{\phi}^{M}=\mathbf{0}$. Imposing zero divergence and integrating (38) in time using the boundary conditions (47), leads to the energy estimate

$$
\begin{aligned}
\left\|\mathbf{u}^{M}\right\|^{2}+\left\|\mathbf{v}^{M}\right\|^{2} & \leq\left\|\mathbf{u}^{M}(t=0)\right\|^{2}+\left\|\mathbf{v}^{M}(t=0)\right\|^{2}+\int_{0}^{T}\left\|\mathbf{g}^{M}\right\|_{\partial D}^{2} d t \\
& -2 \int_{0}^{T}\left\|\mathbf{u}_{x}^{M}\right\|_{\mathbf{A}(\boldsymbol{\mu})}^{2}+\left\|\mathbf{v}_{x}^{M}\right\|_{\mathbf{A}(\boldsymbol{\mu})}^{2}+\left\|\mathbf{u}_{y}^{M}\right\|_{\mathbf{A}(\boldsymbol{\mu})}^{2}+\left\|\mathbf{v}_{y}^{M}\right\|_{\mathbf{A}(\boldsymbol{\mu})}^{2} d t .
\end{aligned}
$$

The energy estimate is the first building block in an analysis of well-posedness. If the problem was linear, the energy estimate would directly lead to uniqueness and by choosing the correct number of boundary conditions, existence would be guaranteed. An energy estimate, uniqueness and existence imply well-posedness for linear problems. For nonlinear problems, the situation is similar but more technically involved. For more details on uniqueness and existence, we refer the reader to [20] where the relations between linear and nonlinear problems are discussed.

Remark 5. The boundary conditions derived above require information about the pressure and the normal component of the velocity at the boundaries. They are not applicable at solid wall boundaries where neither the pressure nor the normal velocity are known. A possible remedy is an extension to the stochastic Galerkin setting of the techniques for dealing with this situation that were investigated in [23, 24] in a deterministic setting.

\section{Numerical Method}

So far, we have derived continuous results for arbitrary spatial domains. Henceforth, for clarity and simplicity we will present the numerical method for the square domain depicted in Figure 1. We introduce a uniform mesh $\left(x_{i}, y_{j}\right)$, for $i=1, \ldots, m_{x}$ and $j=1, \ldots, m_{y}$ on the physical domain $D=$ $[0,2]^{2}$. The grid size is $\Delta x=1 /\left(m_{x}-1\right), \Delta y=1 /\left(m_{y}-1\right)$. 


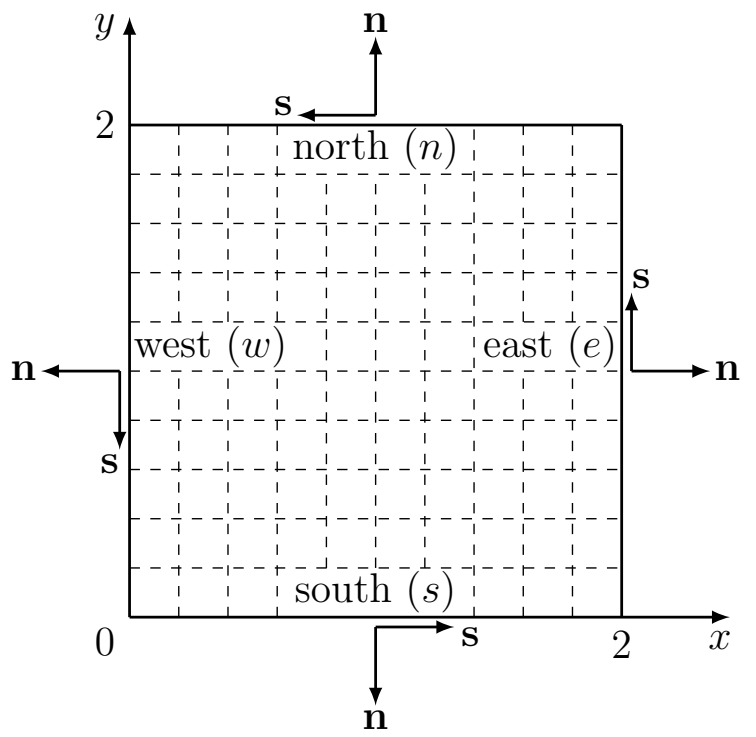

Figure 1: Physical domain $D$ with outward normal (n) and tangent (s) vectors indicated for each boundary $(w, e, s, n)$.

We use finite-difference operators with a summation-by-parts (SBP) property [9] for the semi-discrete problem, i.e., the spatial discretization of the momentum equation (15)-(16) with the time derivative left continuos. The same SBP operators will be used for the discrete update of the pressure field. Summation-by-parts is the discrete equivalent of integration-by-parts, which we frequently used for the continuous energy estimates in Section 4. By mimicking the continuous analysis, we will derive energy estimates for the semi-discrete momentum equations.

The boundary conditions are enforced weakly through a simultaneous approximation term (SAT) technique [12] that penalizes the deviation from the exact boundary data $\mathbf{g}$. The matrices

$$
\mathbf{E}_{m}=\operatorname{diag}(0, \ldots, 0,1), \quad \mathbf{E}_{1}=\operatorname{diag}(1,0, \ldots, 0), \quad m=m_{x}, m_{y},
$$

are used to position the boundary conditions at the boundary nodes of the spatial discretization. 
The first derivative SBP operator was introduced in $[9,10]$ and is approximated by $\mathbf{u}_{x} \approx \mathbf{P}^{-1} \mathbf{Q u}$, where

$$
\mathbf{Q}+\mathbf{Q}^{T}=\operatorname{diag}(-1,0, \ldots, 0,1)=\mathbf{E}_{m}-\mathbf{E}_{1} \equiv \tilde{\mathbf{B}} .
$$

$\mathbf{P}$ is a positive definite diagonal matrix and defines a matrix norm. Operators of order $2 n, n \in \mathbb{N}$, in the interior of the domain are combined with boundary closures of order of accuracy $n$. It is possible to design operators with higher order accuracy at the boundary, but this requires $\mathbf{P}$ to have nonzero off-diagonal entries which can lead to stability problems [25]. We restrict ourselves to diagonal matrices $\mathbf{P}$ since the proofs of stability to be presented later rely on this condition. Subscripts $x$ and $y$ on the operators are used to distinguish between the discretizations in the two coordinate directions, whereas subscripts on vectors denote partial derivatives.

For the approximation of the second derivative SBP operator, introduced in $[26,27]$, we can either use the first derivative operator twice, or use the compact approximation $\mathbf{u}_{x x} \approx \mathbf{P}^{-1}(-\mathbf{M}+\tilde{\mathbf{B D}}) \mathbf{u}$, where $\mathbf{M}+\mathbf{M}^{T} \geq 0, \tilde{\mathbf{B}}$ is given by (48), and $\mathbf{D}$ is a first-derivative approximation at the boundaries, i.e.,

$$
\mathbf{D}=\frac{1}{\Delta x}\left[\begin{array}{ccccc}
d_{1} & d_{2} & d_{3} & \ldots & \\
& 1 & & & \\
& & \ddots & & \\
& & & 1 & \\
& \ldots & -d_{3} & -d_{2} & -d_{1}
\end{array}\right]
$$

Here, $d_{i}, i=1,2,3, \ldots$, are scalar values leading to a consistent firstderivative approximation at the boundaries.

As a convenient computational tool, we will employ the Kronecker product, denoted by $\otimes$ and defined for two matrices $\mathbf{B}$ and $\mathbf{C}$, as

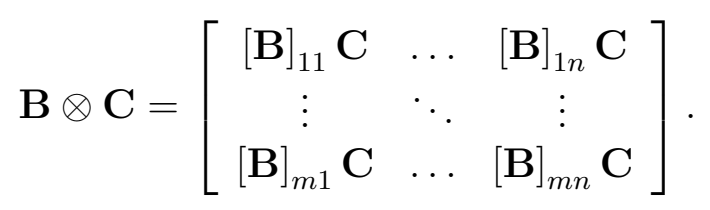

The following notation for the discretization of the derivative operators and matrices of gPC parameters of the momentum and pressure equations is 
used:

$$
\begin{aligned}
& \mathbf{D}_{x}=\mathbf{P}_{x}^{-1} \mathbf{Q}_{x} \otimes \mathbf{I}_{y} \otimes \mathbf{I}^{M}, \\
& \mathbf{D}_{y}=\mathbf{I}_{x} \otimes \mathbf{P}_{y}^{-1} \mathbf{Q}_{y} \otimes \mathbf{I}^{M} \text {, } \\
& \mathbf{D}_{x x}=\mathbf{P}_{x}^{-1}\left(-\mathbf{M}_{x}+\tilde{\mathbf{B}}_{x} \mathbf{D}_{x}\right) \otimes \mathbf{I}_{y} \otimes \mathbf{I}^{M}, \\
& \mathbf{D}_{y y}=\mathbf{I}_{x} \otimes \mathbf{P}_{y}^{-1}\left(-\mathbf{M}_{y}+\tilde{\mathbf{B}}_{y} \mathbf{D}_{y}\right) \otimes \mathbf{I}^{M} \text {, } \\
& \tilde{\mathbf{A}}\left(\mathbf{u}^{M}\right)=\operatorname{diag}\left(\mathbf{A}\left(\mathbf{u}_{i=1, j=1}^{M}\right), \ldots, \mathbf{A}\left(\mathbf{u}_{i=1, j=m_{y}}^{M}\right), \ldots, \mathbf{A}\left(\mathbf{u}_{i=m_{x}, j=m_{y}}^{M}\right)\right) \text {, } \\
& \tilde{\mathbf{A}}\left(\mathbf{v}^{M}\right)=\operatorname{diag}\left(\mathbf{A}\left(\mathbf{v}_{i=1, j=1}^{M}\right), \ldots, \mathbf{A}\left(\mathbf{v}_{i=1, j=m_{y}}^{M}\right), \ldots, \mathbf{A}\left(\mathbf{v}_{i=m_{x}, j=m_{y}}^{M}\right)\right) \text {, } \\
& \tilde{\mathbf{A}}\left(\mathbf{u}_{x}^{M}\right)=\operatorname{diag}\left(\mathbf{A}\left(\left[\mathbf{D}_{x} \mathbf{u}^{M}\right]_{1: M}\right), \ldots, \mathbf{A}\left(\left[\mathbf{D}_{x} \mathbf{u}^{M}\right]_{\left(m_{x}+1\right)\left(m_{y}-1\right)_{M+1} m_{x} m_{y} M}\right)\right) \text {, } \\
& \tilde{\mathbf{A}}\left(\mathbf{v}_{y}^{M}\right)=\operatorname{diag}\left(\mathbf{A}\left(\left[\mathbf{D}_{y} \mathbf{v}^{M}\right]_{1: M}\right), \ldots, \mathbf{A}\left(\left[\mathbf{D}_{y} \mathbf{v}^{M}\right]_{\left(m_{x}+1\right)\left(m_{y}-1\right)_{M+1} m_{x} m_{y} M}\right)\right), \\
& \mathbf{D}_{x}^{(\text {skew })}=\frac{1}{2} \tilde{\mathbf{A}}\left(\mathbf{u}^{M}\right) \mathbf{D}_{x}+\frac{1}{2} \mathbf{D}_{x} \tilde{\mathbf{A}}\left(\mathbf{u}^{M}\right)-\frac{1}{2} \tilde{\mathbf{A}}\left(\mathbf{D}_{x} \mathbf{u}^{M}\right), \\
& \mathbf{D}_{y}^{(\text {skew })}=\frac{1}{2} \tilde{\mathbf{A}}\left(\mathbf{v}^{M}\right) \mathbf{D}_{y}+\frac{1}{2} \mathbf{D}_{y} \tilde{\mathbf{A}}\left(\mathbf{v}^{M}\right)-\frac{1}{2} \tilde{\mathbf{A}}\left(\mathbf{D}_{y} \mathbf{v}^{M}\right), \\
& \tilde{\mathbf{A}}(\boldsymbol{\mu})=\operatorname{diag}\left(\mathbf{A}\left(\boldsymbol{\mu}_{i=1, j=1}\right), \ldots, \mathbf{A}\left(\boldsymbol{\mu}_{i=m_{x}, j=m_{y}}\right)\right),
\end{aligned}
$$

where subscripts $x$ and $y$ denote matrices of size $m_{x}$ and $m_{y}$, respectively. Note that the matrices involving $\tilde{\mathbf{A}}($.$) are block diagonal (but not diagonal).$

\subsection{Discrete Momentum Equations}

The stochastic Galerkin momentum equations (15)-(16) are semi-discretized in space,

$$
\begin{aligned}
\mathbf{u}_{t}^{M}+\mathbf{D}_{x}^{(\text {skew })} \mathbf{u}^{M}+ & \mathbf{D}_{y}^{(\text {skew })} \mathbf{u}^{M}+\mathbf{D}_{x} \mathbf{p}^{M} \\
& =\mathbf{D}_{x} \tilde{\mathbf{A}}(\boldsymbol{\mu}) \mathbf{D}_{x} \mathbf{u}^{M}+\mathbf{D}_{y} \tilde{\mathbf{A}}(\boldsymbol{\mu}) \mathbf{D}_{y} \mathbf{u}^{M}+\mathrm{SAT}^{(\mathbf{u})}, \\
\mathbf{v}_{t}^{M}+\mathbf{D}_{x}^{(\text {skew })} \mathbf{v}^{M}+ & \mathbf{D}_{y}^{(s k e w)} \mathbf{v}^{M}+\mathbf{D}_{y} \mathbf{p}^{M} \\
& =\mathbf{D}_{x} \tilde{\mathbf{A}}(\boldsymbol{\mu}) \mathbf{D}_{x} \mathbf{v}^{M}+\mathbf{D}_{y} \tilde{\mathbf{A}}(\boldsymbol{\mu}) \mathbf{D}_{y} \mathbf{v}^{M}+\mathrm{SAT}^{(\mathbf{v})},
\end{aligned}
$$

where the penalty terms $\mathrm{SAT}^{(\mathbf{u})}$ and $\mathrm{SAT}^{(\mathbf{v})}$ for the boundary conditions will be given below. The application of the first derivative twice for the viscous second derivative terms yields a wide stencil. Alternatively, one may use the second derivative operator for varying coefficients, see [28], to obtain a more narrow stencil. In order to construct discrete boundary conditions for (49) and (50), the block-diagonal eigenvalue decompositions

$$
\tilde{\mathbf{A}}\left(\mathbf{u}^{M}\right)=\mathbf{X}_{\mathbf{u}} \boldsymbol{\Lambda}_{\mathbf{u}} \mathbf{X}_{\mathbf{u}}^{T}, \quad \tilde{\mathbf{A}}\left(\mathbf{v}^{M}\right)=\mathbf{X}_{\mathbf{v}} \boldsymbol{\Lambda}_{\mathbf{v}} \mathbf{X}_{\mathbf{v}}^{T},
$$


are introduced. Let the diagonal matrices containing the eigenvalues (43) and (46) evaluated on the four boundaries $(w, e, s, n)$, be given by

$$
\begin{array}{ll}
\boldsymbol{\Lambda}_{5}^{w}=-\frac{1}{2} \boldsymbol{\Lambda}_{\mathbf{u}}-\left(\frac{1}{4} \boldsymbol{\Lambda}_{\mathbf{u}}^{2}+2 \mathbf{I}^{M}\right)^{1 / 2}, & \boldsymbol{\Lambda}_{5}^{e}=\frac{1}{2} \boldsymbol{\Lambda}_{\mathbf{u}}-\left(\frac{1}{4} \boldsymbol{\Lambda}_{\mathbf{u}}^{2}+2 \mathbf{I}^{M}\right)^{1 / 2}, \\
\boldsymbol{\Lambda}_{5}^{s}=-\frac{1}{2} \boldsymbol{\Lambda}_{\mathbf{v}}-\left(\frac{1}{4} \boldsymbol{\Lambda}_{\mathbf{v}}^{2}+2 \mathbf{I}^{M}\right)^{1 / 2}, & \boldsymbol{\Lambda}_{5}^{n}=\frac{1}{2} \boldsymbol{\Lambda}_{\mathbf{v}}-\left(\frac{1}{4} \boldsymbol{\Lambda}_{\mathbf{v}}^{2}+2 \mathbf{I}^{M}\right)^{1 / 2}, \\
\boldsymbol{\Lambda}_{2}^{w}=-\frac{1}{2} \boldsymbol{\Lambda}_{\mathbf{u}}-\left(\frac{1}{4} \boldsymbol{\Lambda}_{\mathbf{u}}^{2}+\mathbf{I}^{M}\right)^{1 / 2}, & \boldsymbol{\Lambda}_{2}^{e}=\frac{1}{2} \boldsymbol{\Lambda}_{\mathbf{u}}-\left(\frac{1}{4} \boldsymbol{\Lambda}_{\mathbf{u}}^{2}+\mathbf{I}^{M}\right)^{1 / 2} \\
\boldsymbol{\Lambda}_{2}^{s}=-\frac{1}{2} \boldsymbol{\Lambda}_{\mathbf{v}}-\left(\frac{1}{4} \boldsymbol{\Lambda}_{\mathbf{v}}^{2}+\mathbf{I}^{M}\right)^{1 / 2}, & \boldsymbol{\Lambda}_{2}^{n}=\frac{1}{2} \boldsymbol{\Lambda}_{\mathbf{v}}-\left(\frac{1}{4} \boldsymbol{\Lambda}_{\mathbf{v}}^{2}+\mathbf{I}^{M}\right)^{1 / 2}
\end{array}
$$

The difference in sign in the above expressions is due to whether the outward normal vector is aligned with the positive velocity direction or not.

The discrete boundary conditions corresponding to (47) are given by

$$
\begin{aligned}
-\left(\mathbf{X}_{\mathbf{u}} \boldsymbol{\Lambda}_{5} \mathbf{X}_{\mathbf{u}}^{T} \mathbf{u}^{M}\right)_{w}+\mathbf{p}_{w}^{M}-\mathbf{A}(\boldsymbol{\mu})\left(\mathbf{D}_{x} \mathbf{u}^{M}\right)_{w} & =\mathbf{g}_{w}^{(\mathbf{u})} \\
\left(\mathbf{X}_{\mathbf{u}} \boldsymbol{\Lambda}_{5} \mathbf{X}_{\mathbf{u}}^{T} \mathbf{u}^{M}\right)_{e}+\mathbf{p}_{e}^{M}-\mathbf{A}(\boldsymbol{\mu})\left(\mathbf{D}_{x} \mathbf{u}^{M}\right)_{e} & =\mathbf{g}_{e}^{(\mathbf{u})} \\
\left(\mathbf{X}_{\mathbf{v}} \boldsymbol{\Lambda}_{2} \mathbf{X}_{\mathbf{v}}^{T} \mathbf{u}^{M}\right)_{s}+\mathbf{A}(\boldsymbol{\mu})\left(\mathbf{D}_{y} \mathbf{u}^{M}\right)_{s} & =\mathbf{g}_{s}^{(\mathbf{u})} \\
-\left(\mathbf{X}_{\mathbf{v}} \boldsymbol{\Lambda}_{2} \mathbf{X}_{\mathbf{v}}^{T} \mathbf{u}^{M}\right)_{n}+\mathbf{A}(\boldsymbol{\mu})\left(\mathbf{D}_{y} \mathbf{u}^{M}\right)_{n} & =\mathbf{g}_{n}^{(\mathbf{u})} \\
-\left(\mathbf{X}_{\mathbf{u}} \boldsymbol{\Lambda}_{2} \mathbf{X}_{\mathbf{u}}^{T} \mathbf{v}^{M}\right)_{w}-\mathbf{A}(\boldsymbol{\mu})\left(\mathbf{D}_{x} \mathbf{v}^{M}\right)_{w} & =\mathbf{g}_{w}^{(\mathbf{v})} \\
\left(\mathbf{X}_{\mathbf{u}} \boldsymbol{\Lambda}_{2} \mathbf{X}_{\mathbf{u}}^{T} \mathbf{v}^{M}\right)_{e}-\mathbf{A}(\boldsymbol{\mu})\left(\mathbf{D}_{x} \mathbf{v}^{M}\right)_{e} & =\mathbf{g}_{e}^{(\mathbf{v})} \\
-\left(\mathbf{X}_{\mathbf{v}} \boldsymbol{\Lambda}_{5} \mathbf{X}_{\mathbf{v}}^{T} \mathbf{v}^{M}\right)_{s}+\mathbf{p}_{s}^{M}-\mathbf{A}(\boldsymbol{\mu})\left(\mathbf{D}_{y} \mathbf{v}^{M}\right)_{s} & =\mathbf{g}_{s}^{(\mathbf{v})} \\
\left(\mathbf{X}_{\mathbf{v}} \boldsymbol{\Lambda}_{5} \mathbf{X}_{\mathbf{v}}^{T} \mathbf{v}^{M}\right)_{n}+\mathbf{p}_{n}^{M}-\mathbf{A}(\boldsymbol{\mu})\left(\mathbf{D}_{y} \mathbf{v}^{M}\right)_{n} & =\mathbf{g}_{n}^{(\mathbf{v})}
\end{aligned}
$$

for the left, right, lower and upper boundaries respectively. Note the difference in sign in (51)-(58) depending on the orientation of the normal and tangential vectors as displayed in Figure 1.

The boundary conditions are imposed weakly by choosing appropriate penalty coefficients $\tau_{\alpha}^{(\mathbf{w})}(\alpha=w, e, s, n$ and $\mathbf{w}=\mathbf{u}, \mathbf{v})$ on the deviation of the computed boundary values from given boundary data. The SAT for the 
discretized momentum equations (49)-(50) are given by

$$
\begin{aligned}
S A T^{(\mathbf{u})} & =\tau_{w}^{(\mathbf{u})}\left(\mathbf{P}_{x}^{-1} \mathbf{E}_{1} \otimes \mathbf{I}_{y} \otimes \mathbf{I}^{M}\right)\left(-\mathbf{X}_{\mathbf{u}} \Lambda_{5}^{w} \mathbf{X}_{\mathbf{u}}^{T} \mathbf{u}^{M}+\mathbf{p}^{M}-\tilde{\mathbf{A}}(\boldsymbol{\mu}) \mathbf{D}_{x} \mathbf{u}^{M}-\mathbf{g}_{w}^{(\mathbf{u})}\right) \\
& +\tau_{e}^{(\mathbf{u})}\left(\mathbf{P}_{x}^{-1} \mathbf{E}_{m_{x}} \otimes \mathbf{I}_{y} \otimes \mathbf{I}^{M}\right)\left(\mathbf{X}_{\mathbf{u}} \boldsymbol{\Lambda}_{5}^{e} \mathbf{X}_{\mathbf{u}}^{T} \mathbf{u}^{M}+\mathbf{p}^{M}-\tilde{\mathbf{A}}(\boldsymbol{\mu}) \mathbf{D}_{x} \mathbf{u}^{M}-\mathbf{g}_{e}^{(\mathbf{u})}\right) \\
& +\tau_{s}^{(\mathbf{u})}\left(\mathbf{I}_{x} \otimes \mathbf{P}_{y}^{-1} \mathbf{E}_{1} \otimes \mathbf{I}^{M}\right)\left(\mathbf{X}_{\mathbf{v}} \boldsymbol{\Lambda}_{2}^{s} \mathbf{X}_{\mathbf{v}}^{T} \mathbf{u}^{M}+\tilde{\mathbf{A}}(\boldsymbol{\mu}) \mathbf{D}_{y} \mathbf{u}^{M}-\mathbf{g}_{s}^{(\mathbf{u})}\right) \\
& +\tau_{n}^{(\mathbf{u})}\left(\mathbf{I}_{x} \otimes \mathbf{P}_{y}^{-1} \mathbf{E}_{m_{y}} \otimes \mathbf{I}^{M}\right)\left(-\mathbf{X}_{\mathbf{v}} \boldsymbol{\Lambda}_{2}^{n} \mathbf{X}_{\mathbf{v}}^{T} \mathbf{u}^{M}+\tilde{\mathbf{A}}(\boldsymbol{\mu}) \mathbf{D}_{y} \mathbf{u}^{M}-\mathbf{g}_{n}^{(\mathbf{u})}\right), \\
S A T^{(\mathbf{v})} & =\tau_{w}^{(\mathbf{v})}\left(\mathbf{P}_{x}^{-1} \mathbf{E}_{1} \otimes \mathbf{I}_{y} \otimes \mathbf{I}^{M}\right)\left(-\mathbf{X}_{\mathbf{u}} \boldsymbol{\Lambda}_{2}^{w} \mathbf{X}_{\mathbf{u}}^{T} \mathbf{v}^{M}-\tilde{\mathbf{A}}(\boldsymbol{\mu}) \mathbf{D}_{x} \mathbf{v}^{M}-\mathbf{g}_{w}^{(\mathbf{v})}\right) \\
& +\tau_{e}^{(\mathbf{v})}\left(\mathbf{P}_{x}^{-1} \mathbf{E}_{m_{x}} \otimes \mathbf{I}_{y} \otimes \mathbf{I}^{M}\right)\left(\mathbf{X}_{\mathbf{u}} \Lambda_{2}^{e} \mathbf{X}_{\mathbf{u}}^{T} \mathbf{v}^{M}-\tilde{\mathbf{A}}(\boldsymbol{\mu}) \mathbf{D}_{x} \mathbf{v}^{M}-\mathbf{g}_{e}^{(\mathbf{v})}\right) \\
& +\tau_{s}^{(\mathbf{v})}\left(\mathbf{I}_{x} \otimes \mathbf{P}_{y}^{-1} \mathbf{E}_{1} \otimes \mathbf{I}^{M}\right)\left(-\mathbf{X}_{\mathbf{v}} \boldsymbol{\Lambda}_{5}^{s} \mathbf{X}_{\mathbf{v}}^{T} \mathbf{v}^{M}+\mathbf{p}^{M}-\tilde{\mathbf{A}}(\boldsymbol{\mu}) \mathbf{D}_{y} \mathbf{v}^{M}-\mathbf{g}_{s}^{(\mathbf{v})}\right) \\
& +\tau_{n}^{(\mathbf{v})}\left(\mathbf{I}_{x} \otimes \mathbf{P}_{y}^{-1} \mathbf{E}_{m_{y}} \otimes \mathbf{I}^{M}\right)\left(\mathbf{X}_{\mathbf{v}} \boldsymbol{\Lambda}_{5}^{n} \mathbf{X}_{\mathbf{v}}^{T} \mathbf{v}^{M}+\mathbf{p}^{M}-\tilde{\mathbf{A}}(\boldsymbol{\mu}) \mathbf{D}_{y} \mathbf{v}^{M}-\mathbf{g}_{n}^{(\mathbf{v})}\right) .
\end{aligned}
$$

\subsection{The Discretized Poisson Equation for the Pressure}

The momentum equations require the pressure to be known. The SBP discretization of the stochastic Galerkin pressure equation (19) (temporarily ignoring the boundary conditions) is given by

$$
\begin{aligned}
& \left(\mathbf{D}_{x x}+\mathbf{D}_{y y}\right) \mathbf{p}^{M}=\mathbf{D}_{x}\left(\mathbf{A}\left(\boldsymbol{\mu}_{x}\right) \mathbf{D}_{x} \mathbf{u}^{M}\right)+\mathbf{D}_{y}\left(\mathbf{A}\left(\boldsymbol{\mu}_{x}\right) \mathbf{D}_{y} \mathbf{u}^{M}\right)+\mathbf{D}_{x}\left(\mathbf{A}\left(\boldsymbol{\mu}_{y}\right) \mathbf{D}_{x} \mathbf{v}^{M}\right) \\
+ & \mathbf{D}_{y}\left(\mathbf{A}\left(\boldsymbol{\mu}_{y}\right) \mathbf{D}_{y} \mathbf{v}^{M}\right)-\mathbf{A}\left(\mathbf{D}_{x} \mathbf{u}^{M}\right) \mathbf{D}_{x} \mathbf{u}^{M}-2 \mathbf{A}\left(\mathbf{D}_{y} \mathbf{u}^{M}\right) \mathbf{D}_{x} \mathbf{v}^{M}-\mathbf{A}\left(\mathbf{D}_{y} \mathbf{v}^{M}\right) \mathbf{D}_{y} \mathbf{v}^{M} .
\end{aligned}
$$

We use a weak imposition of boundary conditions and solve a linear system of equations for the pressure update in each time-step. The penalty parameters

are chosen to ensure that the resulting matrix operator is non-singular. The boundary conditions are given in the form

$$
\mathbf{p}^{M}=\mathbf{g}_{k}^{(\mathbf{p})}, \quad(x, y) \in \partial D_{k}, \quad k=w, e, s, n .
$$

The boundary data $\mathbf{g}_{k}^{(\mathbf{p})}$ are manufactured by solving (51), (52), (57) and (58) for the pressure and substituting the $\mathbf{D}_{x} \mathbf{u}^{M}$ derivative using the dissipative divergence boundary conditions (23) in discrete form. The result is

$$
\begin{aligned}
& \mathbf{g}_{w}^{(\mathbf{u})}-\mathbf{A}(\boldsymbol{\mu})\left(\mathbf{D}_{y} \mathbf{v}^{M}\right)_{w}+\left(\mathbf{X}_{\mathbf{u}} \boldsymbol{\Lambda}_{5} \mathbf{X}_{\mathbf{u}}^{T} \mathbf{u}^{M}\right)_{w}=\mathbf{g}_{w}^{(\mathbf{p})} \\
& \mathbf{g}_{e}^{(\mathbf{u})}-\mathbf{A}(\boldsymbol{\mu})\left(\mathbf{D}_{y} \mathbf{v}^{M}\right)_{e}-\left(\mathbf{X}_{\mathbf{u}} \boldsymbol{\Lambda}_{5} \mathbf{X}_{\mathbf{u}}^{T} \mathbf{u}^{M}\right)_{e}=\mathbf{g}_{e}^{(\mathbf{p})} \\
& \mathbf{g}_{s}^{(\mathbf{v})}-\mathbf{A}(\boldsymbol{\mu})\left(\mathbf{D}_{x} \mathbf{u}^{M}\right)_{s}+\left(\mathbf{X}_{\mathbf{v}} \boldsymbol{\Lambda}_{5} \mathbf{X}_{\mathbf{v}}^{T} \mathbf{v}^{M}\right)_{s}=\mathbf{g}_{s}^{(\mathbf{p})} \\
& \mathbf{g}_{n}^{(\mathbf{v})}-\mathbf{A}(\boldsymbol{\mu})\left(\mathbf{D}_{x} \mathbf{u}^{M}\right)_{n}-\left(\mathbf{X}_{\mathbf{v}} \boldsymbol{\Lambda}_{5} \mathbf{X}_{\mathbf{v}}^{T} \mathbf{v}^{M}\right)_{n}=\mathbf{g}_{n}^{(\mathbf{p})}
\end{aligned}
$$


Similar to the boundary conditions of the momentum equations, the boundary conditions of the pressure equation are imposed weakly through the use of penalty terms. The penalty coefficients are chosen large enough to make sure that the pressure system matrix is not singular or ill-conditioned. Similar issues in a different context are discussed in [29].

\subsection{Time Stability of the Numerical Method}

To get a semi-discrete energy estimate for the momentum equations, we multiply (49) from the left by $\left(\mathbf{u}^{M}\right)^{T} \overline{\mathbf{P}}$ where $\overline{\mathbf{P}}=\left(\mathbf{P}_{x} \otimes \mathbf{P}_{y} \otimes \mathbf{I}^{M}\right)$, and add the transpose of the result to itself. By using $\overline{\mathbf{P}} \tilde{\mathbf{A}}=\tilde{\mathbf{A}} \overline{\mathbf{P}}$ and the SBP property (48), we get

$$
\begin{aligned}
\frac{d}{d t} & \left\|\mathbf{u}^{M}\right\|_{\overline{\mathbf{P}}}^{2}+ \\
& \underbrace{+\frac{1}{2}\left(\mathbf{u}^{M}\right)^{T} \tilde{\mathbf{A}}\left(\mathbf{u}^{M}\right)\left[\tilde{\mathbf{B}}_{x} \otimes \mathbf{P}_{y} \otimes \mathbf{I}^{M}\right] \mathbf{u}^{M}+\frac{1}{2}\left(\mathbf{u}^{M}\right)^{T}\left[\tilde{\mathbf{B}}_{x} \otimes \mathbf{P}_{y} \otimes \mathbf{I}^{M}\right] \tilde{\mathbf{A}}\left(\mathbf{u}^{M}\right) \mathbf{u}^{M}}
\end{aligned}
$$

Boundary terms from $\mathbf{D}_{x}^{(\text {skew })}$

$$
\underbrace{+\frac{1}{2}\left(\mathbf{u}^{M}\right)^{T} \tilde{\mathbf{A}}\left(\mathbf{v}^{M}\right)\left[\mathbf{P}_{x} \otimes \tilde{\mathbf{B}}_{y} \otimes \mathbf{I}^{M}\right] \mathbf{u}^{M}+\frac{1}{2}\left(\mathbf{u}^{M}\right)^{T}\left[\mathbf{P}_{x} \otimes \tilde{\mathbf{B}}_{y} \otimes \mathbf{I}^{M}\right] \tilde{\mathbf{A}}\left(\mathbf{v}^{M}\right) \mathbf{u}^{M}}
$$

Boundary terms from $\mathbf{D}_{y}^{(\text {skew })}$

$$
\begin{aligned}
& \underbrace{-\left(\mathbf{u}^{M}\right)^{T} \overline{\mathbf{P}}\left(\tilde{\mathbf{A}}\left(\mathbf{u}_{x}^{M}\right)+\tilde{\mathbf{A}}\left(\mathbf{v}_{y}^{M}\right)\right) \mathbf{u}^{M}}_{\text {Residual term }} \underbrace{-2\left(\mathbf{u}^{M}\right)^{T}\left(\mathbf{Q}_{x}^{T} \otimes \mathbf{P}_{y} \otimes \mathbf{I}^{M}\right) \mathbf{p}^{M}}_{\text {Pressure PDE term }} \\
& \text { Residual term } \\
& \underbrace{+2\left(\mathbf{u}^{M}\right)^{T}\left(\mathbf{E}_{m_{x}} \otimes \mathbf{P}_{y} \otimes \mathbf{I}^{M}\right) \mathbf{p}^{M}-2\left(\mathbf{u}^{M}\right)^{T}\left(\mathbf{E}_{1} \otimes \mathbf{P}_{y} \otimes \mathbf{I}^{M}\right) \mathbf{p}^{M}}
\end{aligned}
$$

Pressure boundary terms

$$
\begin{gathered}
=\underbrace{-2\left(\mathbf{u}^{M}\right)^{T}\left(\mathbf{Q}_{x}^{T} \otimes \mathbf{I}_{y} \otimes \mathbf{I}^{M}\right)\left(\mathbf{P}_{x}^{-1} \otimes \mathbf{P}_{y} \otimes \mathbf{I}^{M}\right) \tilde{\mathbf{A}}(\boldsymbol{\mu})\left(\mathbf{Q}_{x} \otimes \mathbf{I}_{y} \otimes \mathbf{I}^{M}\right) \mathbf{u}^{M}}_{\text {Viscous PDE } x \text {-term }} \\
\underbrace{-2\left(\mathbf{u}^{M}\right)^{T}\left(\mathbf{I}_{x} \otimes \mathbf{Q}_{y}^{T} \otimes \mathbf{I}^{M}\right)\left(\mathbf{P}_{x} \otimes \mathbf{P}_{y}^{-1} \otimes \mathbf{I}^{M}\right) \tilde{\mathbf{A}}(\boldsymbol{\mu})\left(\mathbf{I}_{x} \otimes \mathbf{Q}_{y} \otimes \mathbf{I}^{M}\right) \mathbf{u}^{M}}_{\text {Viscous PDE }}
\end{gathered}
$$

Viscous PDE $y$-term

$$
\underbrace{+2\left(\mathbf{u}^{M}\right)^{T}\left[\left(\mathbf{E}_{m_{x}} \otimes \mathbf{P}_{y} \otimes \mathbf{I}^{M}\right)-\left(\mathbf{E}_{1} \otimes \mathbf{P}_{y} \otimes \mathbf{I}^{M}\right)\right] \tilde{\mathbf{A}}(\boldsymbol{\mu}) \mathbf{D}_{x} \mathbf{u}^{M}}_{\text {Viscous boun }}
$$

Viscous boundary terms east and west

$$
\underbrace{+2\left(\mathbf{u}^{M}\right)^{T}\left[\left(\mathbf{P}_{x} \otimes \mathbf{E}_{m_{y}} \otimes \mathbf{I}^{M}\right)-\left(\mathbf{P}_{x} \otimes \mathbf{E}_{1} \otimes \mathbf{I}^{M}\right)\right] \tilde{\mathbf{A}}(\boldsymbol{\mu}) \mathbf{D}_{y} \mathbf{u}^{M}}_{\text {Viscous boundary terms }}+\mathrm{SAT} .
$$

Viscous boundary terms north and south 
We group the terms in (59) and the corresponding expression for $\mathbf{v}^{M}$ into SAT/boundary terms, dissipative terms, and residual terms, and analyze them separately.

\subsubsection{Boundary Terms and SAT}

The matrix $\left(\mathbf{P}_{x}^{-1} \otimes \mathbf{P}_{y} \otimes \mathbf{I}^{M}\right) \tilde{\mathbf{A}}(\boldsymbol{\mu})=\tilde{\mathbf{A}}(\boldsymbol{\mu})\left(\mathbf{P}_{x}^{-1} \otimes \mathbf{P}_{y} \otimes \mathbf{I}^{M}\right)$ is symmetric and positive definite. Using this commutativity property and considering the west boundary and the corresponding SAT with zero data, we have

$$
\begin{gathered}
B T_{w}^{(\mathbf{u})}=\left(\mathbf{u}^{M}\right)^{T}\left(\mathbf{E}_{1} \otimes \mathbf{P}_{y} \otimes \mathbf{I}^{M}\right) \tilde{\mathbf{A}}\left(\mathbf{u}^{M}\right) \mathbf{u}^{M}+2\left(\mathbf{u}^{M}\right)^{T}\left(\mathbf{E}_{1} \otimes \mathbf{P}_{y} \otimes \mathbf{I}^{M}\right) \mathbf{p}^{M} \\
-2\left(\mathbf{u}^{M}\right)^{T}\left(\mathbf{E}_{1} \otimes \mathbf{P}_{y} \otimes \mathbf{I}^{M}\right) \tilde{\mathbf{A}}(\boldsymbol{\mu}) \mathbf{D}_{x} \mathbf{u}^{M} \\
+2 \tau_{w}^{(\mathbf{u})}\left(\mathbf{u}^{M}\right)^{T}\left(\mathbf{E}_{1} \otimes \mathbf{P}_{y} \otimes \mathbf{I}^{M}\right)\left(-\mathbf{X}_{\mathbf{u}} \boldsymbol{\Lambda}_{5}^{w} \mathbf{X}_{\mathbf{u}}^{T} \mathbf{u}^{M}+\mathbf{p}^{M}-\tilde{\mathbf{A}}(\boldsymbol{\mu}) \mathbf{D}_{x} \mathbf{u}^{M}\right) .
\end{gathered}
$$

With the choice $\tau_{w}^{(\mathbf{u})}=-1$, the west boundary terms simplify to

$$
\begin{aligned}
B T_{w}^{(\mathbf{u})}=\left(\mathbf{u}^{M}\right)^{T}\left(\mathbf{E}_{1} \otimes \mathbf{P}_{y} \otimes \mathbf{I}^{M}\right)\left(\tilde{\mathbf{A}}\left(\mathbf{u}^{M}\right)+2 \mathbf{X}_{\mathbf{u}} \boldsymbol{\Lambda}_{5}^{w} \mathbf{X}_{\mathbf{u}}^{T}\right) \mathbf{u}^{M} \\
=-\left(\mathbf{u}^{M}\right)^{T}\left(\mathbf{E}_{1} \otimes \mathbf{P}_{y} \otimes \mathbf{I}^{M}\right) \mathbf{X}_{\mathbf{u}}\left(\boldsymbol{\Lambda}_{\mathbf{u}}^{2}+8 \mathbf{I}\right)^{1 / 2} \mathbf{X}_{\mathbf{u}}^{T} \mathbf{u}^{M},
\end{aligned}
$$

with $-\left(\mathbf{E}_{1} \otimes \mathbf{P}_{y} \otimes \mathbf{I}^{M}\right) \mathbf{X}_{\mathbf{u}}\left(\boldsymbol{\Lambda}_{\mathbf{u}}^{2}+8 \mathbf{I}\right)^{1 / 2} \mathbf{X}_{\mathbf{u}}^{T}$ being negative semi-definite.

The analysis is similar for the remaining three boundaries. For the east boundary and SAT with $\mathbf{g}_{e}=\mathbf{0}$, we obtain

$$
\begin{aligned}
B T_{e}^{(\mathbf{u})}=-\left(\mathbf{u}^{M}\right)^{T}\left(\mathbf{E}_{m_{x}} \otimes \mathbf{P}_{y} \otimes \mathbf{I}^{M}\right) \tilde{\mathbf{A}}\left(\mathbf{u}^{M}\right) \mathbf{u}^{M}-2\left(\mathbf{u}^{M}\right)^{T}\left(\mathbf{E}_{m_{x}} \otimes \mathbf{P}_{y} \otimes \mathbf{I}^{M}\right) \mathbf{p}^{M} \\
+2\left(\mathbf{u}^{M}\right)^{T}\left(\mathbf{E}_{m_{x}} \otimes \mathbf{P}_{y} \otimes \mathbf{I}^{M}\right) \mathbf{A}(\boldsymbol{\mu}) \mathbf{D}_{x} \mathbf{u}^{M} \\
+2 \tau_{e}^{(\mathbf{u})}\left(\mathbf{u}^{M}\right)^{T}\left(\mathbf{E}_{m_{x}} \otimes \mathbf{P}_{y} \otimes \mathbf{I}^{M}\right)\left(\mathbf{X}_{\mathbf{u}} \boldsymbol{\Lambda}_{5}^{e} \mathbf{X}_{\mathbf{u}}^{T} \mathbf{u}^{M}+\mathbf{p}^{M}-\mathbf{A}(\boldsymbol{\mu}) \mathbf{D}_{x} \mathbf{u}^{M}\right)
\end{aligned}
$$

With $\tau_{e}^{(\mathbf{u})}=1$, the east boundary terms become

$$
\begin{aligned}
B T_{e}^{(\mathbf{u})}=\left(\mathbf{u}^{M}\right)^{T}\left(\mathbf{E}_{m_{x}}\right. & \left.\otimes \mathbf{P}_{y} \otimes \mathbf{I}^{M}\right)\left(-\tilde{\mathbf{A}}\left(\mathbf{u}^{M}\right)+2 \mathbf{X}_{\mathbf{u}} \boldsymbol{\Lambda}_{5}^{e} \mathbf{X}_{\mathbf{u}}^{T}\right) \mathbf{u}^{M} \\
& =-\left(\mathbf{u}^{M}\right)^{T}\left(\mathbf{E}_{m_{x}} \otimes \mathbf{P}_{y} \otimes \mathbf{I}^{M}\right) \mathbf{X}_{\mathbf{u}}\left(\Lambda_{\mathbf{u}}^{2}+8 \mathbf{I}\right)^{1 / 2} \mathbf{X}_{\mathbf{u}}^{T} \mathbf{u}^{M}
\end{aligned}
$$

which again is negative semi-definite.

The south and north boundaries involve no pressure terms. With $\tau_{s}^{(\mathbf{u})}=1$, we obtain

$$
B T_{s}^{(\mathbf{u})}=-\left(\mathbf{u}^{M}\right)^{T}\left(\mathbf{P}_{x} \otimes \mathbf{E}_{1} \otimes \mathbf{I}^{M}\right) \mathbf{X}_{\mathbf{v}}\left(\boldsymbol{\Lambda}_{\mathbf{v}}^{2}+4 \mathbf{I}\right)^{1 / 2} \mathbf{X}_{\mathbf{v}}^{T} \mathbf{u}^{M},
$$


and $\tau_{n}^{(\mathbf{u})}=-1$ yields

$$
B T_{n}^{(\mathbf{u})}=-\left(\mathbf{u}^{M}\right)^{T}\left(\mathbf{P}_{x} \otimes \mathbf{E}_{m_{y}} \otimes \mathbf{I}^{M}\right) \mathbf{X}_{\mathbf{v}}\left(\Lambda_{v}^{2}+4 \mathbf{I}\right)^{1 / 2} \mathbf{X}_{\mathbf{v}}^{T} \mathbf{u}^{M} .
$$

We find that the terms at all four boundaries are negative and are generalizations of the deterministic counterparts. The penalty terms are scalars, $\tau_{i}^{(\mathbf{w})}= \pm 1(\mathbf{w}=\mathbf{u}, \mathbf{v}, i=w, e, s, n)$ and thus do not change with the order $M$ of gPC expansion. A similar analysis for the $\mathbf{v}^{M}$ momentum equation (see appendix B) yields $\tau_{w}^{(\mathbf{v})}=\tau_{s}^{(\mathbf{v})}=-1$ and $\tau_{e}^{(\mathbf{v})}=\tau_{n}^{(\mathbf{v})}=1$ for stability.

The boundary terms are collected together and we get

$$
B T \equiv B T_{w}^{(\mathbf{u})}+B T_{e}^{(\mathbf{u})}+B T_{n}^{(\mathbf{u})}+B T_{s}^{(\mathbf{u})}+B T_{w}^{(\mathbf{v})}+B T_{e}^{(\mathbf{v})}+B T_{n}^{(\mathbf{v})}+B T_{s}^{(\mathbf{v})} .
$$

\subsubsection{Residual Terms}

The residual terms

$$
\begin{aligned}
R T \equiv\left(\mathbf{u}^{M}\right)^{T} \overline{\mathbf{P}}\left(\tilde{\mathbf{A}}\left(\mathbf{u}_{x}^{M}\right)+\tilde{\mathbf{A}}\left(\mathbf{v}_{y}^{M}\right)\right) \mathbf{u}^{M}+ & \left(\mathbf{v}^{M}\right)^{T} \overline{\mathbf{P}}\left(\tilde{\mathbf{A}}\left(\mathbf{u}_{x}^{M}\right)+\tilde{\mathbf{A}}\left(\mathbf{v}_{y}^{M}\right)\right) \mathbf{v}^{M} \\
& +2\left(\mathbf{D}_{x} \mathbf{u}^{M}+\mathbf{D}_{y} \mathbf{v}^{M}\right)^{T} \overline{\mathbf{P}} \mathbf{p}^{M}
\end{aligned}
$$

are proportional to the discrete divergence but indefinite. The impact of these terms is assumed to be small, and that will be numerically investigated in Section 6.

\subsubsection{Dissipative Terms}

The viscous PDE terms are

$$
\begin{aligned}
D T \equiv-2\left(\mathbf{D}_{x} \mathbf{u}^{M}\right)^{T} \tilde{\mathbf{A}}(\boldsymbol{\mu}) \overline{\mathbf{P}} \mathbf{D}_{x} \mathbf{u}^{M}-2\left(\mathbf{D}_{y} \mathbf{u}^{M}\right)^{T} \tilde{\mathbf{A}}(\boldsymbol{\mu}) \overline{\mathbf{P}} \mathbf{D}_{y} \mathbf{u}^{M} \\
-2\left(\mathbf{D}_{x} \mathbf{v}^{M}\right)^{T} \tilde{\mathbf{A}}(\boldsymbol{\mu}) \overline{\mathbf{P}} \mathbf{D}_{x} \mathbf{v}^{M}-2\left(\mathbf{D}_{y} \mathbf{v}^{M}\right)^{T} \tilde{\mathbf{A}}(\boldsymbol{\mu}) \overline{\mathbf{P}} \mathbf{D}_{y} \mathbf{v}^{M} .
\end{aligned}
$$

The relation (62) is negative semi-definite if $\tilde{\mathbf{A}}(\boldsymbol{\mu}) \overline{\mathbf{P}}$ is positive definite. $\tilde{\mathbf{A}}(\boldsymbol{\mu}) \overline{\mathbf{P}}$ is block diagonal and thus positive definite if and only if all of its diagonal blocks are positive definite. The matrix block corresponding to the spatial point $\left(x_{i}, y_{j}\right)$ is given by $\left[\mathbf{P}_{x}\right]_{i i}\left[\mathbf{P}_{y}\right]_{j j} \mathbf{A}\left(\boldsymbol{\mu}\left(x_{i}, y_{j}\right)\right)$, for $i=1, \ldots, m_{x}$ and $j=1, \ldots, m_{y}$. The diagonal entries of $\mathbf{P}_{x}$ and $\mathbf{P}_{y}$ are all positive, and $\mathbf{A}\left(\boldsymbol{\mu}\left(x_{i}, y_{j}\right)\right)$ is positive definite for all $\left(x_{i}, y_{j}\right)$. Hence, $\tilde{\mathbf{A}}(\boldsymbol{\mu}) \overline{\mathbf{P}}$ is positive definite.

Assembling the boundary, residual and dissipative terms, the semi-discrete energy estimate becomes

$$
\frac{d}{d t}\left(\left\|\mathbf{u}^{M}\right\|_{\overline{\mathbf{P}}}^{2}+\left\|\mathbf{v}^{M}\right\|_{\overline{\mathbf{P}}}^{2}\right)=B T+R T+D T,
$$


where BT is defined by (60), RT by (61), and DT by (62). The boundary terms $B T$ and the dissipative terms DT are negative and lead to decay of the norm of the velocity. The residual terms $R T$ are proportional to the discrete divergence and expected to be small. Thus, we expect the norm of the discrete velocity to decay over time and the solution to be time-stable. The discrete energy estimate is analogous to the continuous energy estimate (38). It is important to note that the continuous analysis of well-posedness served as a roadmap to obtain a discrete energy estimate by replacing integrationby-parts by summation-by-parts and using discrete versions of the continuous boundary conditions.

\section{Numerical Results}

We will consider two exact solutions to the incompressible Navier-Stokes equations and verify the accuracy of the numerical approximation in the laminar flow regime. We are interested in the numerical convergence of statistical properties $f$ of the solution variables and measure the discrete $\ell_{2}$ errors. We define the error in $f$ as

$$
\epsilon_{\ell_{2}}^{(f)}=\left\|f-f_{r e f}\right\|_{\ell_{2}}=\left(\Delta x \Delta y \sum_{i=1}^{m_{x}} \sum_{j=1}^{m_{y}}\left(f\left(x_{i}, y_{j}, t\right)-f_{r e f}\left(x_{i}, y_{j}, t\right)\right)^{2}\right)^{1 / 2}
$$

where a quantity of interest $f$ can be calculated as a function of the gPC coefficients (for instance, if $f=\operatorname{Var}(u)$, then we use the approximation $\left.f \approx \sum_{k=2}^{M} u_{i}^{2}\right)$. If $f$ is taken to be vector valued, i.e., the vector of gPC coefficients, we first compute the vector 2 -norm of $f$. In that case, $\epsilon_{\ell_{2}}^{(f)}$ denotes a double norm with some abuse of notation. The error in the statistics is due to both the discretization error and the truncation of the gPC series.

\subsection{Taylor Vortex}

Consider the stochastic Taylor vortex model with components exactly satisfying the incompressible Navier-Stokes equations,

$$
\begin{aligned}
& u(x, y, t, \boldsymbol{\xi})=-\cos (\alpha) \sin (\beta) \exp \left(-2 \pi^{2} \mu(\boldsymbol{\xi}) t\right)+u_{\infty} \cos (\theta), \\
& v(x, y, t, \boldsymbol{\xi})=\sin (\alpha) \cos (\beta) \exp \left(-2 \pi^{2} \mu(\boldsymbol{\xi}) t\right)+u_{\infty} \sin (\theta), \\
& p(x, y, t, \boldsymbol{\xi})=-\frac{1}{4}[\cos (2 \alpha)+\cos (2 \beta)] \exp \left(-4 \pi^{2} \mu(\boldsymbol{\xi}) t\right), \\
& \alpha=\pi\left(x-x_{0}-u_{\infty} \cos (\theta) t\right), \quad \beta=\pi\left(y-y_{0}-u_{\infty} \sin (\theta) t\right) .
\end{aligned}
$$


In the numerical experiments we will use $x_{0}=y_{0}=0.5, u_{\infty}=0, \theta=\pi / 4$, and different stochastic models for $\mu$. The gPC coefficients of the exact solution (63) are given by (9)-(11) and the reference solution is calculated through numerical quadrature approximation of the stochastic integrals for the gPC coefficients defined by (2). The analytical solution is deterministic at $t=0$ but stochastic for all $t>0$. Reference values for statistics, e.g. mean and variance, are computed via numerical integration of functions of the analytical solution. Even though the test case has a periodic solution, we use the more general non-periodic boundary conditions derived previously.

The number of gPC coefficients needed to obtain a given accuracy of the solution is in general not known in advance. We will use relatively loworder gPC expansions $(M=3,4)$ of Legendre polynomials for the numerical experiments. For the Taylor vortex problem, the gPC coefficients can be calculated up to the accuracy of the numerical quadrature rule. Figure 2 shows the decay of the gPC coefficients, measured as

$$
\left\|u_{k}\right\|_{\ell_{2}}=\left(\Delta x \Delta y \sum_{i=1}^{m_{x}} \sum_{j=1}^{m_{y}}\left(u_{k}\left(x_{i}, y_{j}, t\right)\right)^{2}\right)^{1 / 2},
$$

for $k=1, \ldots, 6$, evaluated at $t=0.2$. It was found that the grid size $(\Delta x, \Delta y)$ had an insignificant effect on the norm of the gPC coefficients. The GaussLegendre quadrature rule integrates up to tenth-order polynomials exactly. Most of the variance is captured by 3-4 gPC terms due to the fast decay of the coefficients. 


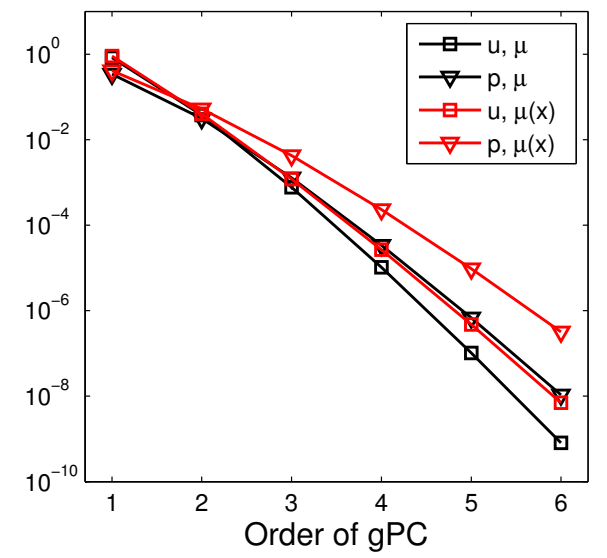

Figure 2: Decay of the gPC coefficients of $u$ and $p$ for the Taylor vortex problem. Constant $\mu \sim U[0.03,0.07]$ and spatially varying $\mu(\mathbf{x}) \sim U[0.005,0.05]$.

\subsubsection{Spatially uniform $\mu$}

We assume $\mu=0.05+0.02 \xi, \xi \sim U[-1,1]$ and compare the results to the deterministic case $\mu=0.05$. The gPC coefficients decay quickly for this diffusive problem. The low-order expansion $M=3$ leads to sufficiently good stochastic resolution in the sense that the error from the spatial discretization is dominant and we may check the spatial order of convergence by numerical experiments. The means and standard deviations of the stochastic solution are shown in Figure 3. 

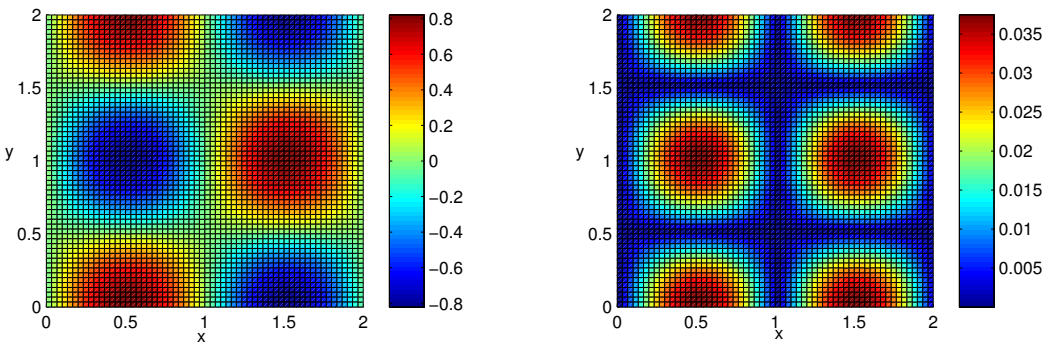

(a) Mean (left) and standard deviation (right) of velocity component $u$.
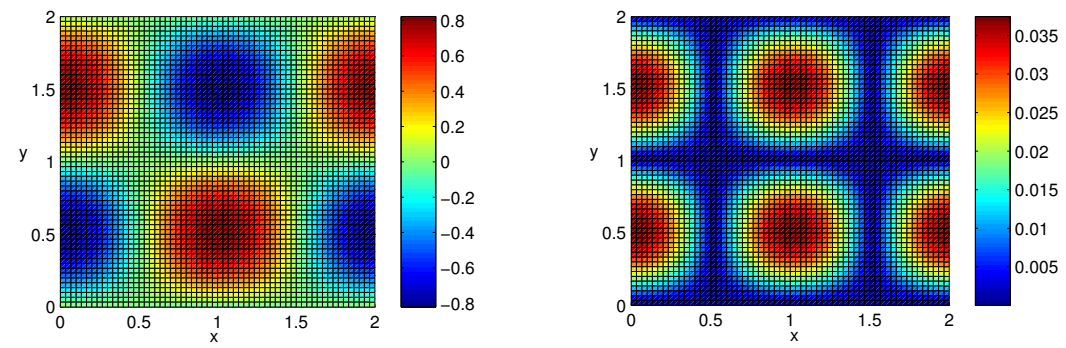

(b) Mean (left) and standard deviation (right) of velocity component $v$.
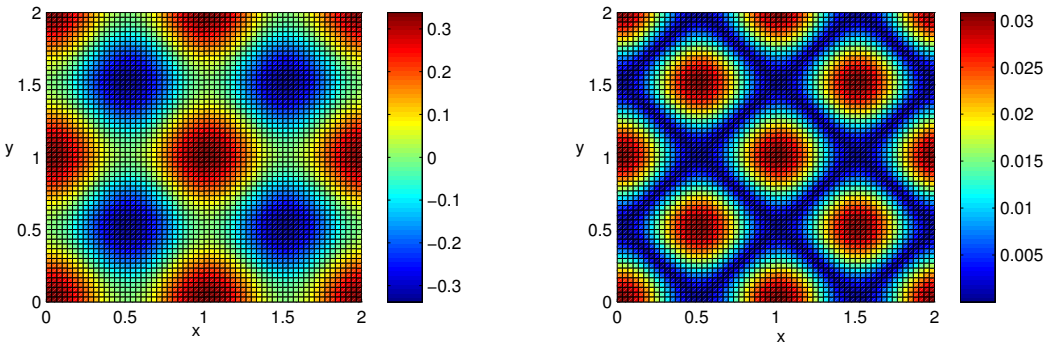

(c) Mean (left) and standard deviation (right) of pressure $p$.

Figure 3: Means and standard deviations at $t=0.2, m_{x}=m_{y}=121$, order of $\mathrm{gPC} M=3$, and $\mu \sim U[0.03,0.07]$.

Table 1 displays the spatial convergence with mesh refinement for the deterministic case. Table 2 shows the convergence for the stochastic Galerkin formulation of the stochastic problem with $M=3 \mathrm{gPC}$ terms. A fourthorder accurate Runge-Kutta method is used for the time integration and the temporal error is negligible compared to the spatial error. We use spatial SBP operators with 8th order of accuracy in the interior and 4th order of accuracy on the boundary. According to the analysis in [30], we may expect 
5th order of accuracy globally for the incompressible Navier-Stokes equations and the given choice of spatial operators. The numerical results indicate a slightly higher order of accuracy.

\begin{tabular}{ccccccc}
\hline$\Delta x, \Delta y$ & $\epsilon_{\ell_{2}}^{(\mathbf{u})}$ & $q^{(\mathbf{u})}$ & $\epsilon_{\ell_{2}}^{(\mathbf{p})}$ & $q^{(\mathbf{p})}$ & $\epsilon_{\ell_{2}}^{(\boldsymbol{\phi})}$ & $q^{(\boldsymbol{\phi})}$ \\
\hline \hline $2 / 60$ & $2.2 \cdot 10^{-5}$ & 5.9 & $1.1 \cdot 10^{-4}$ & 5.7 & $3.7 \cdot 10^{-4}$ & \\
$2 / 80$ & $4.1 \cdot 10^{-6}$ & 5.9 & $2.1 \cdot 10^{-5}$ & 5.8 & $6.4 \cdot 10^{-5}$ & 6.1 \\
$2 / 100$ & $1.1 \cdot 10^{-6}$ & 5.9 & $5.8 \cdot 10^{-5}$ & 5.9 & $1.6 \cdot 10^{-5}$ & 6.2 \\
$2 / 120$ & $3.7 \cdot 10^{-7}$ & 5.9 & $2.0 \cdot 10^{-6}$ & 5.9 & $5.2 \cdot 10^{-6}$ & 6.2 \\
$2 / 140$ & $1.5 \cdot 10^{-7}$ & & $7.9 \cdot 10^{-7}$ & & $2.0 \cdot 10^{-6}$ & 6.2 \\
\hline
\end{tabular}

Table 1: Numerical convergence for the deterministic vortex problem, $t=0.2$. $\ell_{2}$ error $(\epsilon)$ and order of convergence $(q)$.

We investigate the errors in expectations, standard deviations and the vectors of gPC cofficients. The results for the different statistics are not independent but provide more accessible information. The error in the standard deviation is affected by the truncation error resulting from truncation of the infinite sum in (3) to $M$ terms. For statistics that are independent of the truncated terms, the truncation error will still have some impact on the remaining terms since the truncation implies that we solve a modified problem. The lower-order terms are expected to be less affected by the stochastic truncation. With sufficient stochastic resolution, we can thus expect the error in the expectation (i.e., the first gPC coefficient) to be dominated by the spatial discretization error. Thus, we expect the observed rate of convergence for the expectations in Table 2 to be the most accurate indicator of the actual spatial order of convergence since the other statistics are somewhat polluted by the stochastic truncation error. Note also the remarkably high order of convergence for the velocity divergence (which consists of derivatives) in Table 1 and 2. 


\begin{tabular}{|c|c|c|c|c|c|c|}
\hline$\Delta x, \Delta y$ & $\epsilon_{\ell_{2}}^{(\mathbb{E}(u))}$ & $q^{(\mathbb{E}(u))}$ & $\epsilon_{\ell_{2}}^{(\operatorname{Std}(u))}$ & $q^{(\operatorname{Std}(u))}$ & $\epsilon_{\ell_{2}}^{(\mathbf{u})}$ & $q^{(\mathbf{u})}$ \\
\hline $\begin{array}{c}2 / 40 \\
2 / 60 \\
2 / 80 \\
2 / 100 \\
2 / 120\end{array}$ & $\begin{array}{l}2.2 \cdot 10^{-4} \\
2.2 \cdot 10^{-5} \\
4.1 \cdot 10^{-6} \\
1.1 \cdot 10^{-6} \\
3.8 \cdot 10^{-7}\end{array}$ & $\begin{array}{l}5.6 \\
5.9 \\
5.9 \\
5.8\end{array}$ & $\begin{array}{l}3.2 \cdot 10^{-5} \\
3.5 \cdot 10^{-6} \\
6.1 \cdot 10^{-7} \\
1.5 \cdot 10^{-7} \\
5.1 \cdot 10^{-8}\end{array}$ & $\begin{array}{l}5.5 \\
6.1 \\
6.2 \\
6.1\end{array}$ & $\begin{array}{l}2.2 \cdot 10^{-4} \\
2.2 \cdot 10^{-5} \\
4.2 \cdot 10^{-6} \\
1.2 \cdot 10^{-6} \\
4.0 \cdot 10^{-7}\end{array}$ & $\begin{array}{l}5.6 \\
5.9 \\
5.9 \\
5.7\end{array}$ \\
\hline$\Delta x, \Delta y$ & $\epsilon_{\ell_{2}}^{(\mathbb{E}(p))}$ & $q^{(\mathbb{E}(p))}$ & $\epsilon_{\ell_{2}}^{(\operatorname{Std}(p))}$ & $q^{(S t d(p))}$ & $\epsilon_{\ell_{2}}^{(\mathbf{p})}$ & $q^{(\mathbf{p})}$ \\
\hline $\begin{array}{c}2 / 40 \\
2 / 60 \\
2 / 80 \\
2 / 100 \\
2 / 120\end{array}$ & $\begin{array}{l}7.5 \cdot 10^{-4} \\
7.9 \cdot 10^{-5} \\
1.5 \cdot 10^{-5} \\
4.0 \cdot 10^{-6} \\
1.4 \cdot 10^{-6}\end{array}$ & $\begin{array}{l}5.6 \\
5.8 \\
5.9 \\
5.9\end{array}$ & $\begin{array}{l}7.2 \cdot 10^{-5} \\
7.0 \cdot 10^{-6} \\
1.3 \cdot 10^{-6} \\
3.4 \cdot 10^{-7} \\
1.4 \cdot 10^{-7}\end{array}$ & $\begin{array}{l}5.8 \\
6.0 \\
5.9 \\
5.0\end{array}$ & $\begin{array}{l}7.5 \cdot 10^{-4} \\
7.9 \cdot 10^{-5} \\
1.5 \cdot 10^{-5} \\
4.1 \cdot 10^{-6} \\
1.5 \cdot 10^{-6}\end{array}$ & $\begin{array}{l}5.6 \\
5.8 \\
5.8 \\
5.6\end{array}$ \\
\hline$\Delta x, \Delta y$ & $\epsilon_{\ell_{2}}^{(\mathbb{E}(\phi))}$ & $q^{(\mathbb{E}(\phi))}$ & $\epsilon_{\ell_{2}}^{(\operatorname{Std}(\phi))}$ & $q^{(\operatorname{Std}(\phi))}$ & $\epsilon_{\ell_{2}}^{(\phi)}$ & $q^{(\phi)}$ \\
\hline $\begin{array}{c}2 / 40 \\
2 / 60 \\
2 / 80 \\
2 / 100 \\
2 / 120\end{array}$ & $\begin{array}{l}4.0 \cdot 10^{-3} \\
3.9 \cdot 10^{-4} \\
6.8 \cdot 10^{-5} \\
1.7 \cdot 10^{-5} \\
5.6 \cdot 10^{-6}\end{array}$ & $\begin{array}{l}5.7 \\
6.1 \\
6.2 \\
6.2\end{array}$ & $\begin{array}{l}1.1 \cdot 10^{-3} \\
1.2 \cdot 10^{-4} \\
2.1 \cdot 10^{-5} \\
5.2 \cdot 10^{-6} \\
1.7 \cdot 10^{-6}\end{array}$ & $\begin{array}{l}5.5 \\
6.1 \\
6.3 \\
6.3\end{array}$ & $\begin{array}{l}4.1 \cdot 10^{-3} \\
4.1 \cdot 10^{-4} \\
7.2 \cdot 10^{-5} \\
1.8 \cdot 10^{-5} \\
5.8 \cdot 10^{-6}\end{array}$ & $\begin{array}{l}5.7 \\
6.1 \\
6.2 \\
6.2\end{array}$ \\
\hline
\end{tabular}

Table 2: Numerical convergence for the stochastic vortex problem, $M=3$, $t=0.2$. Error and order of convergence $q$ for mean value $(\mathbb{E}()$.$) , standard$ deviation $(\operatorname{Std}()$.$) , and the vector of \mathrm{gPC}$ coefficients $(\mathbf{u} / \mathbf{p} / \boldsymbol{\phi})$.

\subsubsection{Spatially varying $\mu$}

To investigate the performance of the present formulation for problems with spatially varying viscosity, we next consider the solution to (4)-(6), where the viscosity is a random field $\mu(\mathbf{x}, \boldsymbol{\xi})=\bar{\mu}+\sigma_{\mu} k^{-1 / 2} \cos (k \pi x) \cos (k \pi y) \xi$. We set $\bar{\mu}=0.0275$, and $\sigma_{\mu}=0.0225$ (i.e., $\mu$ varies in the interval $[0.005,0.05]$ ). Using the method of manufactured solutions [31,32], (4)-(5) and (8) are modified by adding appropriate source terms to satisfy the solution (63). Results on spatial convergence are shown in Table 3.

Remark 6. In the numerical experiments, $\mu$ is one-dimensional in stochas- 
tic space and we set $k=1$, but the extension to multiple random variables is straightforward by using a series of terms with different integer $k$. This implies a Karhunen-Loève-like expansion with, respectively, eigenvalues and eigenfunctions of the form $k^{-1}$ and $\cos (k \pi x) \cos (k \pi y)$ for the covariance function of $\mu(\mathbf{x}, \boldsymbol{\xi})$.

\begin{tabular}{|c|c|c|c|c|c|c|}
\hline$\Delta x, \Delta y$ & $\epsilon_{\ell_{2}}^{(\mathbb{E}(u))}$ & $q^{(\mathbb{E}(u))}$ & $\epsilon_{\ell_{2}}^{(S t d(u))}$ & $q^{(S t d(u))}$ & $\epsilon_{\ell_{2}}^{(\mathbf{u})}$ & $q^{(\mathbf{u})}$ \\
\hline $\begin{array}{c}2 / 40 \\
2 / 60 \\
2 / 80 \\
2 / 100 \\
2 / 120\end{array}$ & $\begin{array}{l}3.1 \cdot 10^{-4} \\
3.0 \cdot 10^{-5} \\
5.5 \cdot 10^{-6} \\
1.5 \cdot 10^{-6} \\
5.1 \cdot 10^{-7}\end{array}$ & $\begin{array}{l}5.7 \\
5.9 \\
5.9 \\
5.9\end{array}$ & $\begin{array}{l}7.8 \cdot 10^{-5} \\
1.3 \cdot 10^{-5} \\
3.1 \cdot 10^{-6} \\
9.7 \cdot 10^{-7} \\
3.8 \cdot 10^{-7}\end{array}$ & $\begin{array}{l}4.5 \\
5.0 \\
5.2 \\
5.2\end{array}$ & $\begin{array}{l}3.2 \cdot 10^{-4} \\
3.3 \cdot 10^{-5} \\
6.4 \cdot 10^{-6} \\
1.8 \cdot 10^{-6} \\
6.5 \cdot 10^{-7}\end{array}$ & $\begin{array}{l}5.6 \\
5.7 \\
5.7 \\
5.6\end{array}$ \\
\hline$\Delta x, \Delta y$ & $\epsilon_{\ell_{2}}^{(\mathbb{E}(p))}$ & $q^{(\mathbb{E}(p))}$ & $\epsilon_{\ell_{2}}^{(S t d(p))}$ & $q^{(S t d(p))}$ & $\epsilon_{\ell_{2}}^{(\mathbf{p})}$ & $q^{(\mathbf{p})}$ \\
\hline $\begin{array}{c}2 / 40 \\
2 / 60 \\
2 / 80 \\
2 / 100 \\
2 / 120\end{array}$ & $\begin{array}{l}9.0 \cdot 10^{-4} \\
9.8 \cdot 10^{-5} \\
1.9 \cdot 10^{-5} \\
5.0 \cdot 10^{-6} \\
1.7 \cdot 10^{-6}\end{array}$ & $\begin{array}{l}5.5 \\
5.8 \\
5.9 \\
5.9\end{array}$ & $\begin{array}{l}1.5 \cdot 10^{-4} \\
2.2 \cdot 10^{-5} \\
4.8 \cdot 10^{-6} \\
1.4 \cdot 10^{-6} \\
5.1 \cdot 10^{-7}\end{array}$ & $\begin{array}{l}4.7 \\
5.3 \\
5.5 \\
5.5\end{array}$ & $\begin{array}{l}9.1 \cdot 10^{-4} \\
1.0 \cdot 10^{-4} \\
1.9 \cdot 10^{-5} \\
5.2 \cdot 10^{-6} \\
1.8 \cdot 10^{-6}\end{array}$ & $\begin{array}{l}5.4 \\
5.7 \\
5.8 \\
5.9\end{array}$ \\
\hline$\Delta x, \Delta y$ & $\epsilon_{\ell_{2}}^{(\mathbb{E}(\phi))}$ & $q^{(\mathbb{E}(\phi))}$ & $\epsilon_{\ell_{2}}^{(\operatorname{Std}(\phi))}$ & $q^{(S t d(\phi))}$ & $\epsilon_{\ell_{2}}^{(\boldsymbol{\phi})}$ & $q^{(\phi)}$ \\
\hline $\begin{array}{c}2 / 40 \\
2 / 60 \\
2 / 80 \\
2 / 100 \\
2 / 120\end{array}$ & $\begin{array}{l}7.3 \cdot 10^{-3} \\
7.9 \cdot 10^{-4} \\
1.4 \cdot 10^{-4} \\
3.8 \cdot 10^{-5} \\
1.3 \cdot 10^{-5}\end{array}$ & $\begin{array}{l}5.5 \\
5.9 \\
6.0 \\
6.1\end{array}$ & $\begin{array}{l}1.8 \cdot 10^{-3} \\
3.0 \cdot 10^{-4} \\
6.9 \cdot 10^{-5} \\
2.1 \cdot 10^{-5} \\
7.5 \cdot 10^{-6}\end{array}$ & $\begin{array}{l}4.5 \\
5.1 \\
5.4 \\
5.6\end{array}$ & $\begin{array}{l}7.5 \cdot 10^{-3} \\
8.4 \cdot 10^{-4} \\
1.6 \cdot 10^{-4} \\
4.3 \cdot 10^{-5} \\
1.5 \cdot 10^{-5}\end{array}$ & $\begin{array}{l}5.4 \\
5.8 \\
5.9 \\
6.0\end{array}$ \\
\hline
\end{tabular}

Table 3: Numerical convergence for the stochastic vortex problem, $M=4$, $t=0.2$. Error and order of convergence for mean value $(\mathbb{E}()$.$) , standard$ deviation $(\operatorname{Std}()$.$) , and the vector of \mathrm{gPC}$ coefficients $(\mathbf{u} / \mathbf{p} / \boldsymbol{\phi}) . q$ denotes the observed order of convergence.

The decay of the velocity divergence with mesh refinement verifies that the residual terms (61) proportional to the discrete divergence are indeed 
small. As in the case of spatially uniform $\mu$, remarkably high-order of convergence is observed for the velocity divergence also for the case of spatially varying $\mu$.

Depending on $\mu$, the time-step in the fourth-order Runge-Kutta method is either limited by a factor proportional to $\min (\Delta x, \Delta y)$ or $\min \left(\Delta x^{2}, \Delta y^{2}\right)$. For complex problems and in particular nonlinear problems, exact limits based on analysis are not feasible to obtain. For the numerical results, we have used $\Delta t=0.05 \mathrm{~min}(\Delta x, \Delta y)$. Numerical investigation of the maximum time-step suggests that $\Delta t=0.05 \mathrm{~min}(\Delta x, \Delta y)$ is very close to the time-step limit for the finest meshes, and a factor 4-5 smaller than the time-step limit for the coarsest mesh. When the time-step is further increased, the solutions blow up. The higher-order gPC coefficients are small in magnitude (they decay with the order of gPC) but appear to add sensitivity to the timestep [18]. It is not surprising that the stochastic setting makes the time-step limit more restrictive since the stochastic Galerkin method gives a solution that represents a range of possible values and the most restrictive case will determine the constraint.

\subsection{Poiseuille Flow}

We next consider laminar flow between two plates where the fluid is initially at rest, $u=v=0$. A constant pressure gradient is applied in the horizontal direction with a parabolic inflow profile at the west boundary. The steady state solution with unit density and deterministic boundary conditions is the parabolic Poiseuille profile,

$$
\begin{aligned}
& u(x, y, t, \boldsymbol{\xi})=u_{\infty}\left(1-(y-1)^{2}\right), \\
& v(x, y, t, \boldsymbol{\xi})=0 \\
& p(x, y, t, \boldsymbol{\xi})=1-2 u_{\infty} \mu(\boldsymbol{\xi}) x .
\end{aligned}
$$

As in the previous test case, we use the boundary conditions for the momentum and pressure equations derived in Sections 5.1 and 5.2. Both the form of the boundary conditions and the choice of penalty parameters are the same as in the vortex problem. The only difference is the boundary data. At all boundaries, we impose the data that are obtained by differentiating the steady state solution.

Figure 4 shows the determinitic solution at $t=10$ on a coarse mesh, $m_{x}=m_{y}=24$. The numerical solution is close to the steady state solution and the difference is on the order $10^{-6}$ for both the solution variables and 
the divergence. For this problem, the boundary data and the initial function are not consistent and the divergence is initially non-zero but converges to zero with time. The decay of the velocity divergence with time, even with inconsistent initial data, verifies that the residual terms (61) proportional to the discrete divergence are indeed small. As before, we do not impose zero divergence strongly in the interior but provide dissipative boundary data. As a result, the error in the divergence has been reduced by seven orders of magnitude at $t=10$ for the coarse mesh problem of Figure 4 .

For the stochastic Poiseuille flow with $\mu=0.05+0.02 \xi, \xi \sim U[-1,1]$, we observe a similar convergence to a divergence-free solution, but for the case $M=3$, the error is an order of magnitude larger. The errors in the standard deviation of the solution variables and the divergence are shown in Figure 5. The decay of the velocity divergence over time is shown in Figure 6 for two different mesh sizes, $\Delta x=\Delta y=0.1$ and $\Delta x=\Delta y=0.05$. The decay in the norm of all the included gPC coefficients $\epsilon_{\ell_{2}}^{(\phi)}$ is plotted as a function of time and we see that the stochastic divergence converges to zero at steady-state, despite the inconsistent initial and boundary data. 


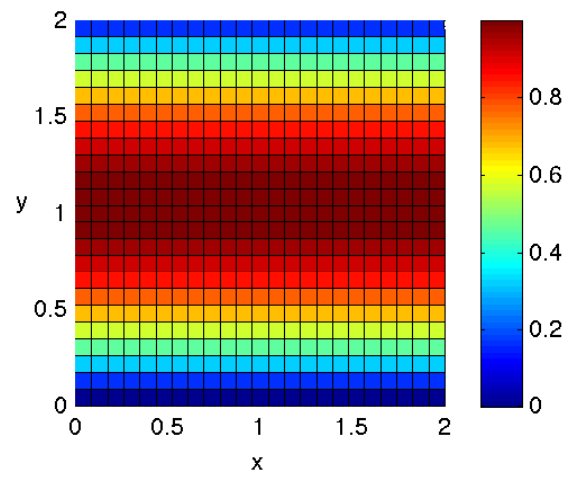

(a) Velocity component $u$.

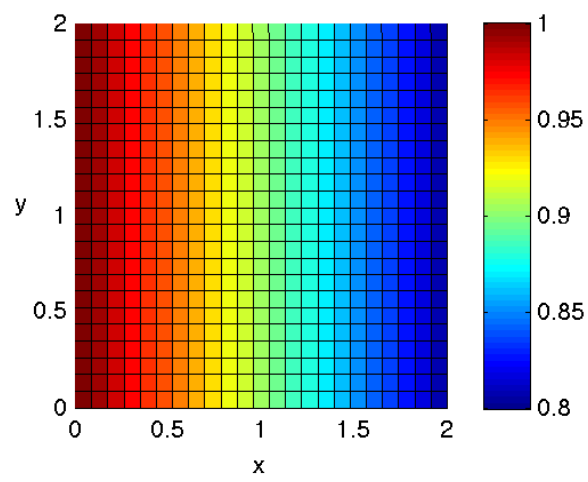

(c) Pressure $p$.

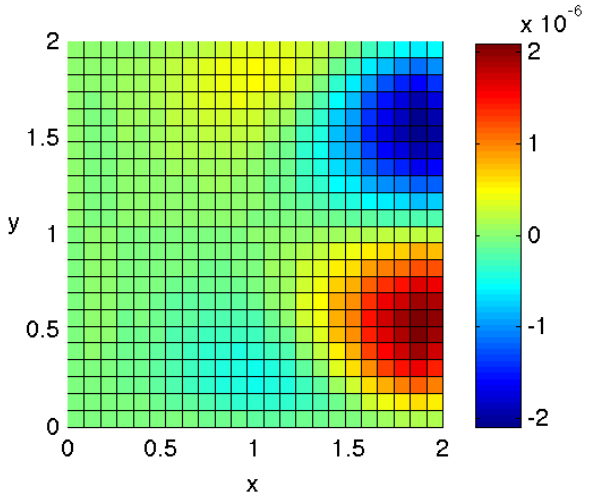

(b) Velocity component $v$.

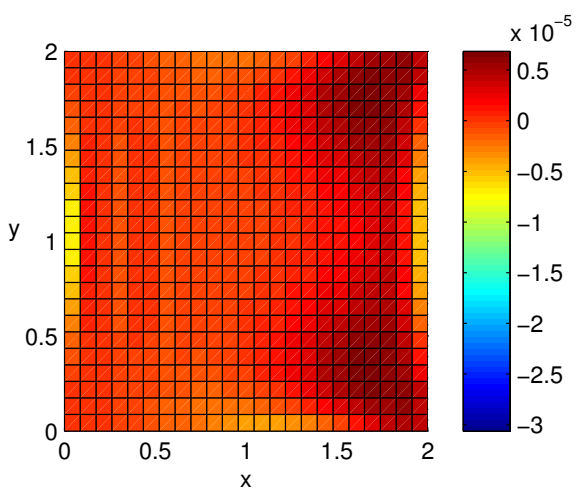

(d) Divergence $\phi$.

Figure 4: Deterministic Poiseuille solution at $t=10, m_{x}=m_{y}=24$. 


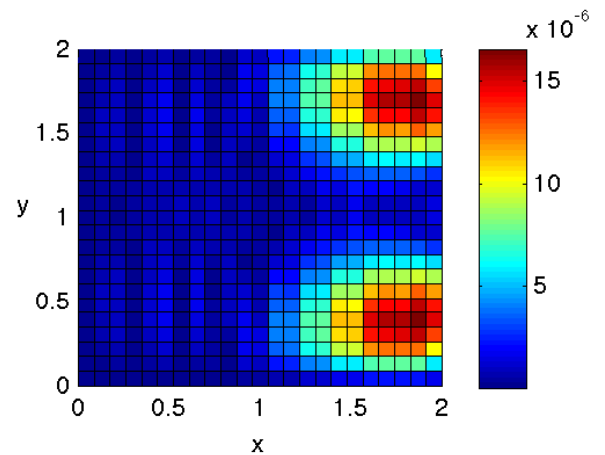

(a) Velocity component $u$.

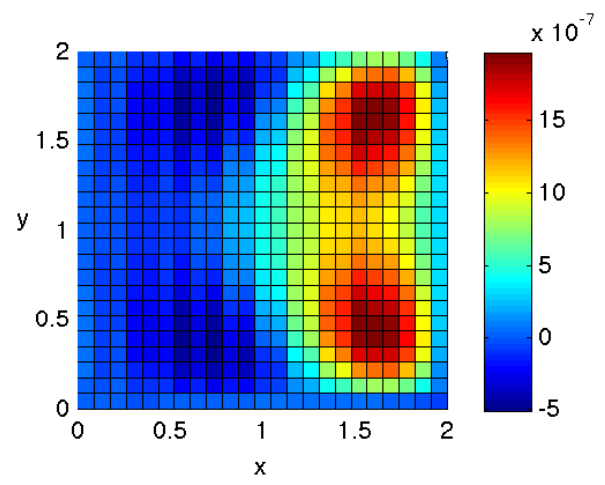

(c) Pressure $p$.

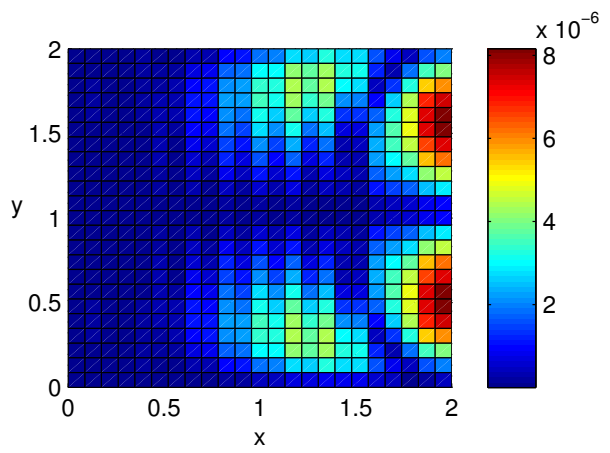

(b) Velocity component $v$.

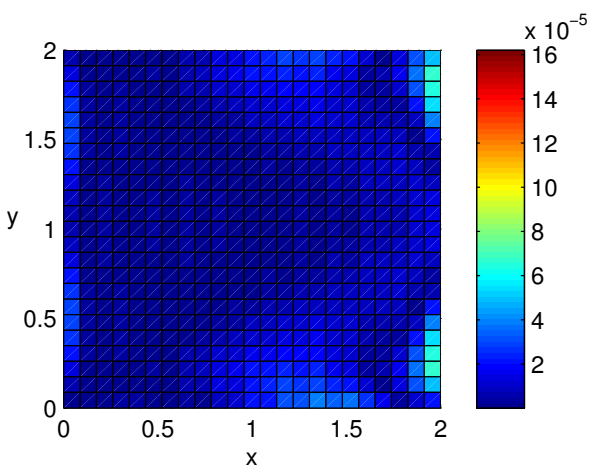

(d) Divergence $\phi$.

Figure 5: Error in the standard deviations for Poiseuille flow at $t=10$, $m_{x}=m_{y}=24$. Stochastic $\mu=0.05+0.02 \xi, \xi \sim U[-1,1]$. 




Figure 6: Decay of the discrete $\ell_{2}$ norm of the gPC coefficients of the velocity divergence for the Poiseuille flow problem over time. For each spatial point we compute the $L_{2}$ norm of the local vector of gPC coefficients. Constant $\mu \sim U[0.03,0.07]$.

The Poiseuille flow problem and the Taylor vortex problem are complementary. The initial velocity field of the Poiseuille flow problem is inconsistent with the boundary data, which leads to relatively large values of the divergence field. Asymptotically, the numerical solution converges to the true steady-state solution with zero velocity divergence. For the Taylor vortex problem, we provided consistent initial data and boundary data satisfying the zero divergence condition. In that case, we could verify high-order spatial convergence for any given time.

The computational cost of this method is similar to that of standard intrusive stochastic Galerkin methods where the system grows linearly in $M$. With a total of $m=m_{x} m_{y}$ grid points, solving the pressure equation amounts to solving a linear $m M \times m M$ system. This is currently done with the standard backslash operator in Matlab, which selects from a set of different methods depending on the pressure system matrix properties. Since the pressure system matrix varies due to the varying boundary conditions, this seemed like an overall more efficient choice compared to finding a linear solver that were optimal for a given set of boundary conditions, but with unknown performance for varying matrix properties.

The time integration of the momentum equtions amounts to computing vector-matrix multiplications of the full system size $(m M \times m M)$. For the boundary conditions, point-wise (in space/time) eigenvalue decompositions 
of size $M \times M$ are performed. For problems where $M$ is chosen large in comparison to $m$, this might be dominating the computational cost.

\section{Conclusions}

We have presented a numerical method for the stochastic Galerkin formulation of the incompressible Navier-Stokes equations that enforces incompressibility weakly. The analysis relies on the properties of summation-byparts operators that are available for different orders of accuracy. These operators all have the same matrix form, so the analysis holds for various orders of accuracy. The result is a stable and high-order accurate numerical method where the divergence converges to zero according to the approximation order of the scheme.

Stability of numerical methods is a prerequisite for convergence to the true solution, but non-trivial to ensure for non-linear problems. For stochastic Galerkin formulations, the situation is further complicated and choosing boundary conditions based on physical arguments may not be sufficient to ensure a numerically stable method. The boundary conditions are stochastic, non-periodic in space, and time-dependent. The number of boundary conditions depends on the order of the gPC expansion, but does not change over time.

In order to derive a stable scheme for the semi-discrete stochastic Galerkin incompressible Navier-Stokes system, the continuous formulation and the deterministic scheme have served as a roadmap. A stable stochastic Galerkin method is highly non-trivial to find without first performing the analysis for the deterministic and continuous problems. Once the appropriate approximate, general continuous formulation and the numerical method have been found, the remaining penalty parameters that lead to stability are straightforward to choose.

The method presented here leads to high-order accuracy for all variables including the velocity divergence. The high-order convergence for the divergence is notable since it would normally be lower for quantities that are derivative approximations of the solution variables. However, similar higherorder numerical convergence for the divergence using finite difference methods has actually been previously reported in, e.g., [23]. Numerical results are presented with discretization operators of eighth-order accuracy in the interior spatial domain, and fourth-order accuracy on the boundaries. With 
a sufficiently accurate stochastic representation, we observe fifth-order global convergence.

\section{Acknowledgements}

The authors would like to thank Anna Nissen for analysis of stability leading to the choice of penalty parameters for the pressure updates. The first author was funded by SUPRI-B at Stanford University and Uni Research, Norway. This material is based upon work of Alireza Doostan supported by the U.S. Department of Energy Office of Science, Office of Advanced Scientific Computing Research, under Award Number DE-SC0006402.

\section{Appendix A Alternative Boundary Conditions}

In order to arrive at an alternative and more dissipative energy estimate for the norm of the divergence coefficients, the boundary integral terms in (20) will be rewritten in a form that allows us to identify growth terms, which can then be controlled by subscription of boundary conditions. Assuming that $\mathbf{A}\left(\mathbf{u}_{\mathbf{n}}^{M}\right)$ is invertible everywhere along the boundary $\partial D$ implies that the boundary terms in (20) can be rewritten as

$$
\begin{aligned}
\left(\boldsymbol{\phi}^{M}\right)^{T} \mathbf{A}\left(\mathbf{u}_{\mathbf{n}}^{M}\right) \boldsymbol{\phi}^{M}-2\left(\boldsymbol{\phi}^{M}\right)^{T} \mathbf{A}(\boldsymbol{\mu}) \frac{\partial \boldsymbol{\phi}^{M}}{\partial \mathbf{n}} & {\left[\mathbf{A}\left(\mathbf{u}_{\mathbf{n}}^{M}\right) \boldsymbol{\phi}^{M}-\mathbf{A}(\boldsymbol{\mu}) \frac{\partial \boldsymbol{\phi}^{M}}{\partial \mathbf{n}}\right]^{T}\left[\mathbf{A}\left(\mathbf{u}_{\mathbf{n}}^{M}\right)\right]^{-1}\left[\mathbf{A}\left(\mathbf{u}_{\mathbf{n}}^{M}\right) \boldsymbol{\phi}^{M}-\mathbf{A}(\boldsymbol{\mu}) \frac{\partial \boldsymbol{\phi}^{M}}{\partial \mathbf{n}}\right] } \\
- & {\left[\mathbf{A}(\boldsymbol{\mu}) \frac{\partial \boldsymbol{\phi}^{M}}{\partial \mathbf{n}}\right]^{T}\left[\mathbf{A}\left(\mathbf{u}_{\mathbf{n}}^{M}\right)\right]^{-1}\left[\mathbf{A}(\boldsymbol{\mu}) \frac{\partial \boldsymbol{\phi}^{M}}{\partial \mathbf{n}}\right] . }
\end{aligned}
$$

Let the eigenvalue decomposition $\left[\mathbf{A}\left(\mathbf{u}_{\mathbf{n}}^{M}\right)\right]^{-1}=\mathbf{X} \boldsymbol{\Lambda}^{-1} \mathbf{X}^{T}$ be given, where $\mathbf{X}$ and $\boldsymbol{\Lambda}^{-1}$ may both vary in space. Then the boundary terms are given by

$$
\begin{array}{r}
{\left[\mathbf{X}^{T}\left(\mathbf{A}\left(\mathbf{u}_{\mathbf{n}}^{M}\right) \boldsymbol{\phi}^{M}-\mathbf{A}(\boldsymbol{\mu}) \frac{\partial \boldsymbol{\phi}^{M}}{\partial \mathbf{n}}\right)\right]^{T} \boldsymbol{\Lambda}^{-1}\left[\mathbf{X}^{T}\left(\mathbf{A}\left(\mathbf{u}_{\mathbf{n}}^{M}\right) \boldsymbol{\phi}^{M}-\mathbf{A}(\boldsymbol{\mu}) \frac{\partial \boldsymbol{\phi}^{M}}{\partial \mathbf{n}}\right)\right]} \\
-\left[\mathbf{X}^{T} \mathbf{A}(\boldsymbol{\mu}) \frac{\partial \boldsymbol{\phi}^{M}}{\partial \mathbf{n}}\right]^{T} \boldsymbol{\Lambda}^{-1}\left[\mathbf{X}^{T} \mathbf{A}(\boldsymbol{\mu}) \frac{\partial \boldsymbol{\phi}^{M}}{\partial \mathbf{n}}\right] .
\end{array}
$$


To get a bound on (A.1), we assign boundary conditions to prescribe the growth terms,

$$
\left.\begin{array}{ll}
\mathbf{X}_{j}^{T}\left(\mathbf{A}\left(\mathbf{u}_{\mathbf{n}}^{M}\right) \boldsymbol{\phi}^{M}-\mathbf{A}(\boldsymbol{\mu}) \frac{\partial \phi^{M}}{\partial \mathbf{n}}\right) & \text { if }[\boldsymbol{\Lambda}]_{j j}<0 \\
\mathbf{X}_{j}^{T} \mathbf{A}(\boldsymbol{\mu}) \frac{\partial \boldsymbol{\phi}^{M}}{\partial \mathbf{n}} & \text { if }[\boldsymbol{\Lambda}]_{j j} \geq 0
\end{array}\right\}=\mathbf{g}_{j}
$$

for $j=1, \ldots, M$ and at each point on the boundary. The vector $\mathbf{g}$ contains the boundary data. By using (A.3) in (A.2), we get the estimate

$$
\begin{aligned}
& -\oint_{\partial D}\left(\mathbf{u}_{\mathbf{n}}^{M}\right)^{T} \mathbf{A}\left(\boldsymbol{\phi}^{M}\right) \boldsymbol{\phi}^{M}-2\left(\boldsymbol{\phi}^{M}\right)^{T} \mathbf{A}(\boldsymbol{\mu}) \frac{\partial \boldsymbol{\phi}^{M}}{\partial \mathbf{n}} d s \\
& \leq \oint_{\partial D} \mathbf{g}^{T}\left|\boldsymbol{\Lambda}^{-1}\right| \mathbf{g}-\left[\mathbf{X}^{T} \mathbf{A}\left(\mathbf{u}_{\mathbf{n}}^{M}\right) \boldsymbol{\phi}^{M}-\mathbf{g}\right]^{T}\left|\boldsymbol{\Lambda}^{-1}\right|\left[\mathbf{X}^{T} \mathbf{A}\left(\mathbf{u}_{\mathbf{n}}^{M}\right) \boldsymbol{\phi}^{M}-\mathbf{g}\right] d s
\end{aligned}
$$

for the boundary integral in (20).

Remark 7. If $\mathbf{A}\left(\mathbf{u}_{\mathbf{n}}^{M}\right)$ is not invertible but has at least one nonzero eigenvalue, we may write its eigenvalue decomposition as

$$
\mathbf{A}\left(\mathbf{u}_{\mathbf{n}}^{M}\right)=\left[\begin{array}{ll}
\mathbf{X} & \mathbf{X}_{0}
\end{array}\right]\left[\begin{array}{ll}
\boldsymbol{\Lambda} & \mathbf{0} \\
\mathbf{0} & \mathbf{0}
\end{array}\right]\left[\begin{array}{l}
\mathbf{X}^{T} \\
\mathbf{X}_{0}^{T}
\end{array}\right]
$$

where $\mathbf{X}$ contains the basis vectors corresponding to the nonzero eigenvalues $(\boldsymbol{\Lambda})$ and $\mathbf{X}_{0}$ contains the vectors of the nullspace. Then, since this implies $\mathbf{A}\left(\mathbf{u}_{\mathbf{n}}^{M}\right)=\mathbf{X} \mathbf{\Lambda} \mathbf{X}^{T}$, we may use the latter reduced eigenvalue decomposition and proceed as indicated above to prescribe boundary conditions and obtain an estimate for the boundary integral terms.

Consider sufficiently short times $t \in\left[0, T_{0}\right]$ and let $\delta=\max _{t, x} \operatorname{eig}\left(\mathbf{A}\left(\boldsymbol{\phi}^{M}\right)\right)$. Inserting the estimates (A.4) and (25) into (20), multiplying by the factor $e^{-(\delta+\eta) t}$ and integrating over time from 0 to $T_{0}$, we obtain the energy estimate

$$
\begin{aligned}
&\left\|\boldsymbol{\phi}^{M}\right\|^{2}+\int_{0}^{T_{0}} {\left[2\left\|\boldsymbol{\phi}_{x}^{M}\right\|_{\mathbf{A}(\boldsymbol{\mu})}^{2}+2\left\|\boldsymbol{\phi}_{y}^{M}\right\|_{\mathbf{A}(\boldsymbol{\mu})}^{2}\right] e^{-(\delta+\eta)\left(t-T_{0}\right)} d t } \\
&+\int_{0}^{T_{0}}\left\|\mathbf{X}^{T} \mathbf{A}\left(\mathbf{u}_{n}^{M}\right) \boldsymbol{\phi}^{M}-\mathbf{g}\right\|_{\left|\boldsymbol{\Lambda}^{-1}\right|}^{2} e^{-(\delta+\eta)\left(t-T_{0}\right)} d t \\
& \leq\left\|\boldsymbol{\phi}^{M}(t=0)\right\|^{2} e^{(\delta+\eta) T_{0}}+\int_{0}^{T_{0}}\left[\|\mathbf{g}\|_{\left|\boldsymbol{\Lambda}^{-1}\right|}^{2}+\frac{1}{\eta}\left\|\mathbf{F}^{M}\right\|^{2}\right] e^{-(\delta+\eta)\left(t-T_{0}\right)} d t
\end{aligned}
$$


where $\|\cdot\|_{\left|\boldsymbol{\Lambda}^{-1}\right|}$ is the vector 2 -norm weighted by $\left|\boldsymbol{\Lambda}^{-1}\right|$. We impose zero initial and boundary data, $\boldsymbol{\phi}^{M}(t=0)=\mathbf{g}=\mathbf{0}$, and require that $\mathbf{F}^{M}=\mathbf{0}$. This results in $\left\|\phi^{M}\left(t=T_{0}\right)\right\|^{2} \leq\left\|\phi^{M}(t=0)\right\|^{2}$. Then the argument is repeated for a new time interval $\left[T_{0}, 2 T_{0}\right]$, and we obtain $\left\|\phi^{M}\left(t=2 T_{0}\right)\right\|^{2} \leq\left\|\phi^{M}(t=0)\right\|^{2}$. Iterating this argument for sufficiently short time intervals, we conclude that $\phi^{M}=0$ for all times. Thus, the zero divergence condition is satisfied for all times. 


\section{Appendix B Stability Analysis}

\section{B.1 Energy estimate for the $\mathbf{v}^{M}$ momentum equation}

The energy estimate for $\mathbf{v}^{M}$ is analogous to the energy estimate for $\mathbf{u}^{M}$. Multiply (50) from the left by $\left(\mathbf{v}^{M}\right)^{T} \overline{\mathbf{P}}$ to obtain

$$
\begin{aligned}
& \frac{d}{d t}\left\|\mathbf{v}^{M}\right\|_{\overline{\mathbf{P}}}^{2}+ \\
& \underbrace{+\frac{1}{2}\left(\mathbf{v}^{M}\right)^{T} \tilde{\mathbf{A}}\left(\mathbf{u}^{M}\right)\left[\tilde{\mathbf{B}}_{x} \otimes \mathbf{P}_{y} \otimes \mathbf{I}^{M}\right] \mathbf{v}^{M}+\frac{1}{2}\left(\mathbf{v}^{M}\right)^{T}\left[\tilde{\mathbf{B}}_{x} \otimes \mathbf{P}_{y} \otimes \mathbf{I}^{M}\right] \tilde{\mathbf{A}}\left(\mathbf{u}^{M}\right) \mathbf{v}^{M}} \\
& \text { Boundary terms from } \mathbf{D}_{x}^{(\text {skew })} \\
& \underbrace{+\frac{1}{2}\left(\mathbf{v}^{M}\right)^{T} \tilde{\mathbf{A}}\left(\mathbf{v}^{M}\right)\left[\mathbf{P}_{x} \otimes \tilde{\mathbf{B}}_{y} \otimes \mathbf{I}^{M}\right] \mathbf{v}^{M}+\frac{1}{2}\left(\mathbf{v}^{M}\right)^{T}\left[\mathbf{P}_{x} \otimes \tilde{\mathbf{B}}_{y} \otimes \mathbf{I}^{M}\right] \tilde{\mathbf{A}}\left(\mathbf{v}^{M}\right) \mathbf{v}^{M}} \\
& \text { Boundary terms from } \mathbf{D}_{y}^{(\text {skew })} \\
& \underbrace{-\left(\mathbf{v}^{M}\right)^{T} \overline{\mathbf{P}}\left(\tilde{\mathbf{A}}\left(\mathbf{u}_{x}^{M}\right)+\tilde{\mathbf{A}}\left(\mathbf{v}_{y}^{M}\right)\right) \mathbf{v}^{M}}_{\text {Residual term }} \underbrace{-2\left(\mathbf{v}^{M}\right)^{T}\left(\mathbf{P}_{x} \otimes \mathbf{Q}_{y}^{T} \otimes \mathbf{I}^{M}\right) \mathbf{p}^{M}}_{\text {Pressure PDE term }} \\
& \underbrace{+2\left(\mathbf{v}^{M}\right)^{T}\left(\mathbf{P}_{x} \otimes \mathbf{E}_{m_{y}} \otimes \mathbf{I}^{M}\right) \mathbf{p}^{M}-2\left(\mathbf{v}^{M}\right)^{T}\left(\mathbf{P}_{x} \otimes \mathbf{E}_{1} \otimes \mathbf{I}^{M}\right) \mathbf{p}^{M}} \\
& \text { Pressure boundary terms } \\
& =\underbrace{-2\left(\mathbf{v}^{M}\right)^{T}\left(\mathbf{Q}_{x}^{T} \otimes \mathbf{I}_{y} \otimes \mathbf{I}^{M}\right)\left(\mathbf{P}_{x}^{-1} \otimes \mathbf{P}_{y} \otimes \mathbf{I}^{M}\right) \tilde{\mathbf{A}}(\boldsymbol{\mu})\left(\mathbf{Q}_{x} \otimes \mathbf{I}_{y} \otimes \mathbf{I}^{M}\right) \mathbf{v}^{M}}_{\text {Viscous PDE } x \text {-term }} \\
& \underbrace{-2\left(\mathbf{v}^{M}\right)^{T}\left(\mathbf{I}_{x} \otimes \mathbf{Q}_{y}^{T} \otimes \mathbf{I}^{M}\right)\left(\mathbf{P}_{x} \otimes \mathbf{P}_{y}^{-1} \otimes \mathbf{I}^{M}\right) \tilde{\mathbf{A}}(\boldsymbol{\mu})\left(\mathbf{I}_{x} \otimes \mathbf{Q}_{y} \otimes \mathbf{I}^{M}\right) \mathbf{v}^{M}} \\
& \text { Viscous PDE } y \text {-term } \\
& \underbrace{+2\left(\mathbf{v}^{M}\right)^{T}\left[\left(\mathbf{E}_{m_{x}} \otimes \mathbf{P}_{y} \otimes \mathbf{I}^{M}\right)-\left(\mathbf{E}_{1} \otimes \mathbf{P}_{y} \otimes \mathbf{I}^{M}\right)\right] \tilde{\mathbf{A}}(\boldsymbol{\mu}) \mathbf{D}_{x} \mathbf{v}^{M}} \\
& \text { Viscous boundary terms east and west } \\
& \underbrace{+2\left(\mathbf{v}^{M}\right)^{T}\left[\left(\mathbf{P}_{x} \otimes \mathbf{E}_{m_{y}} \otimes \mathbf{I}^{M}\right)-\left(\mathbf{P}_{x} \otimes \mathbf{E}_{1} \otimes \mathbf{I}^{M}\right)\right] \tilde{\mathbf{A}}(\boldsymbol{\mu}) \mathbf{D}_{y} \mathbf{v}^{M}}_{\text {Viscous boundary terms north and south }}+\mathrm{SAT} .
\end{aligned}
$$


Set $\mathbf{g}_{w}^{(\mathbf{v})}=\mathbf{g}_{e}^{(\mathbf{v})}=\mathbf{g}_{s}^{(\mathbf{v})}=\mathbf{g}_{n}^{(\mathbf{v})}=\mathbf{0}$, and collect the terms boundary-wise. For the west boundary, we have

$$
\begin{aligned}
B T_{w}^{(\mathbf{v})} & =\left(\mathbf{v}^{M}\right)^{T}\left(\mathbf{E}_{1} \otimes \mathbf{P}_{y} \otimes \mathbf{I}^{M}\right) \tilde{\mathbf{A}}\left(\mathbf{u}^{M}\right) \mathbf{v}^{M} \\
-2\left(\mathbf{v}^{M}\right)^{T}\left(\mathbf{E}_{1} \otimes \mathbf{P}_{y} \otimes \mathbf{I}^{M}\right) \tilde{\mathbf{A}}(\boldsymbol{\mu}) \mathbf{D}_{x} \mathbf{v}^{M}+2 \tau_{w}^{(\mathbf{v})}\left(\mathbf{v}^{M}\right)^{T}\left(\mathbf{E}_{1} \otimes \mathbf{P}_{y} \otimes \mathbf{I}^{M}\right) \times & \\
& \times\left(-\mathbf{X}_{\mathbf{u}}\left[-\frac{1}{2} \boldsymbol{\Lambda}_{\mathbf{u}}-\left(\frac{1}{4} \boldsymbol{\Lambda}_{\mathbf{u}}^{2}+\mathbf{I}\right)^{1 / 2}\right] \mathbf{X}_{\mathbf{u}}^{T} \mathbf{v}^{M}-\tilde{\mathbf{A}}(\boldsymbol{\mu}) \mathbf{D}_{x} \mathbf{v}^{M}\right) .
\end{aligned}
$$

With the choice $\tau_{w}^{(\mathbf{v})}=-1,($ B.2) simplifies to

$$
B T_{w}^{(\mathbf{v})}=-\left(\mathbf{v}^{M}\right)^{T}\left(\mathbf{E}_{1} \otimes \mathbf{P}_{y} \otimes \mathbf{I}^{M}\right) \mathbf{X}_{\mathbf{u}}\left(\boldsymbol{\Lambda}_{\mathbf{u}}^{2}+4 \mathbf{I}\right)^{1 / 2} \mathbf{X}_{\mathbf{u}}^{T} \mathbf{v}^{M}
$$

For the east boundary,

$$
\begin{aligned}
B T_{e}^{(\mathbf{v})}=-\left(\mathbf{v}^{M}\right)^{T} & \left(\mathbf{E}_{m_{x}} \otimes \mathbf{P}_{y} \otimes \mathbf{I}^{M}\right) \tilde{\mathbf{A}}\left(\mathbf{u}^{M}\right) \mathbf{v}^{M} \\
+2\left(\mathbf{v}^{M}\right)^{T}\left(\mathbf{E}_{m_{x}}\right. & \left.\otimes \mathbf{P}_{y} \otimes \mathbf{I}^{M}\right) \tilde{\mathbf{A}}(\boldsymbol{\mu}) \mathbf{D}_{x} \mathbf{v}^{M}+2 \tau_{e}^{(\mathbf{v})}\left(\mathbf{v}^{M}\right)^{T}\left(\mathbf{E}_{m_{x}} \otimes \mathbf{P}_{y} \otimes \mathbf{I}^{M}\right) \times \\
& \times\left(\mathbf{X}_{\mathbf{u}}\left[\frac{1}{2} \boldsymbol{\Lambda}_{\mathbf{u}}-\left(\frac{1}{4} \boldsymbol{\Lambda}_{\mathbf{u}}^{2}+\mathbf{I}\right)^{1 / 2}\right] \mathbf{X}_{\mathbf{u}}^{T} \mathbf{v}^{M}-\tilde{\mathbf{A}}(\boldsymbol{\mu}) \mathbf{D}_{x} \mathbf{v}^{M}\right)
\end{aligned}
$$

which simplifies to

$$
B T_{e}^{(\mathbf{v})}=-\left(\mathbf{v}^{M}\right)^{T}\left(\mathbf{E}_{m_{x}} \otimes \mathbf{P}_{y} \otimes \mathbf{I}^{M}\right) \mathbf{X}_{\mathbf{u}}\left(\boldsymbol{\Lambda}_{\mathbf{u}}^{2}+4 \mathbf{I}\right)^{1 / 2} \mathbf{X}_{\mathbf{u}}^{T} \mathbf{v}^{M}
$$

with $\tau_{e}^{(\mathbf{v})}=1$.

The south boundary terms are given by

$$
\begin{aligned}
B T_{s}^{(\mathbf{v})} & =\left(\mathbf{v}^{M}\right)^{T}\left(\mathbf{P}_{x} \otimes \mathbf{E}_{1} \otimes \mathbf{I}^{M}\right) \tilde{\mathbf{A}}\left(\mathbf{v}^{M}\right) \mathbf{v}^{M} \\
+2\left(\mathbf{v}^{M}\right)^{T} & \left(\mathbf{P}_{x} \otimes \mathbf{E}_{1} \otimes \mathbf{I}^{M}\right)\left[\mathbf{p}^{M}-\tilde{\mathbf{A}}(\boldsymbol{\mu}) \mathbf{D}_{y} \mathbf{v}^{M}\right]+2 \tau_{s}^{(\mathbf{v})}\left(\mathbf{v}^{M}\right)^{T}\left(\mathbf{P}_{x} \otimes \mathbf{E}_{1} \otimes \mathbf{I}^{M}\right) \times \\
& \times\left(-\mathbf{X}_{\mathbf{v}}\left[-\frac{1}{2} \boldsymbol{\Lambda}_{\mathbf{v}}-\left(\frac{1}{4} \boldsymbol{\Lambda}_{\mathbf{v}}^{2}+2 \mathbf{I}\right)^{1 / 2}\right] \mathbf{X}_{\mathbf{v}}^{T} \mathbf{v}^{M}+\mathbf{p}^{M}-\tilde{\mathbf{A}}(\boldsymbol{\mu}) \mathbf{D}_{y} \mathbf{v}^{M}\right),
\end{aligned}
$$

which for $\tau_{s}^{(\mathbf{v})}=-1$ becomes

$$
B T_{s}^{(\mathbf{v})}=-\left(\mathbf{v}^{M}\right)^{T}\left(\mathbf{P}_{x} \otimes \mathbf{E}_{1} \otimes \mathbf{I}\right) \mathbf{X}_{\mathbf{v}}\left(\Lambda_{\mathbf{v}}^{2}+8 \mathbf{I}\right)^{1 / 2} \mathbf{X}_{\mathbf{v}}^{T} \mathbf{v}^{M}
$$


Finally, the north boundary terms are

$$
\begin{aligned}
B T_{n}^{(\mathbf{v})}= & -\left(\mathbf{v}^{M}\right)^{T}\left(\mathbf{P}_{x} \otimes \mathbf{E}_{m_{y}} \otimes \mathbf{I}^{M}\right) \tilde{\mathbf{A}}\left(\mathbf{v}^{M}\right) \mathbf{v}^{M} \\
+2\left(\mathbf{v}^{M}\right)^{T} & \left(\mathbf{P}_{x} \otimes \mathbf{E}_{m_{y}} \otimes \mathbf{I}^{M}\right)\left[-\mathbf{p}^{M}+\tilde{\mathbf{A}}(\boldsymbol{\mu}) \mathbf{D}_{y} \mathbf{v}^{M}\right]+2 \tau_{n}^{(\mathbf{v})}\left(\mathbf{v}^{M}\right)^{T}\left(\mathbf{P}_{x} \otimes \mathbf{E}_{m_{y}} \otimes \mathbf{I}^{M}\right) \times \\
& \times\left(\mathbf{X}_{\mathbf{v}}\left[-\frac{1}{2} \boldsymbol{\Lambda}_{\mathbf{v}}-\left(\frac{1}{4} \boldsymbol{\Lambda}_{\mathbf{v}}^{2}+2 \mathbf{I}\right)^{1 / 2}\right] \mathbf{X}_{\mathbf{v}}^{T} \mathbf{v}^{M}+\mathbf{p}^{M}-\tilde{\mathbf{A}}(\boldsymbol{\mu}) \mathbf{D}_{y} \mathbf{v}^{M}\right) .
\end{aligned}
$$

Setting $\tau_{n}^{(\mathbf{v})}=1$ yields

$$
B T_{n}^{(\mathbf{v})}=-\left(\mathbf{v}^{M}\right)^{T}\left(\mathbf{P}_{x} \otimes \mathbf{E}_{m_{y}} \otimes \mathbf{I}^{M}\right) \mathbf{X}_{\mathbf{v}}\left(\Lambda_{\mathbf{v}}^{2}+8 \mathbf{I}\right)^{1 / 2} \mathbf{X}_{\mathbf{v}}^{T} \mathbf{v}^{M}
$$

\section{References}

[1] O. P. Le Maître, O. M. Knio, Spectral Methods for Uncertainty Quantification, 1st Edition, Springer, 2010.

[2] R. G. Ghanem, P. D. Spanos, Stochastic finite elements: a spectral approach, Springer-Verlag, New York, 1991.

[3] D. Xiu, Numerical Methods for Stochastic Computations: A Spectral Method Approach, Princeton University Press, 2010.

[4] O. P. Le Maître, O. M. Knio, H. N. Najm, R. G. Ghanem, A stochastic projection method for fluid flow: I. basic formulation, J. Comput. Phys. 173 (2) (2001) 481-511. doi:10.1006/jcph.2001.6889.

[5] O. P. Le Maître, M. T. Reagan, H. N. Najm, R. G. Ghanem, O. M. Knio, A stochastic projection method for fluid flow: II. random process, J. Comput. Phys. 181 (1) (2002) 9-44. doi:10.1006/jcph.2002.7104.

[6] D. Xiu, G. E. Karniadakis, Modeling uncertainty in flow simulations via generalized polynomial chaos, J. Comput. Phys. 187 (1) (2003) 137-167. doi:10.1016/S0021-9991(03)00092-5.

[7] D. Xiu, D. Lucor, C. H. Su, G. E. Karniadakis, Stochastic modeling of Flow-Structure interactions using generalized polynomial chaos, J. Fluids Eng. 124 (1) (2002) 51-59. doi:10.1115/1.1436089. 
[8] O. P. Le Maître, M. T. Reagan, B. J. Debusschere, H. N. Najm, R. G. Ghanem, O. M. Knio, Natural convection in a closed cavity under stochastic non-Boussinesq conditions., SIAM J. Scientific Computing 26 (2) (2004) 375-394. doi:10.1137/S1064827503422853.

[9] H.-O. Kreiss, G. Scherer, Finite element and finite difference methods for hyperbolic partial differential equations, in: Mathematical Aspects of Finite Elements in Partial Differential Equations, Academic Press, New York, 1974, pp. 179-183.

[10] B. Strand, Summation by parts for finite difference approximations for d/dx, J. Comput. Phys. 110 (1) (1994) 47-67. doi:10.1006/jcph.1994.1005.

[11] B. Gustafsson, H.-O. Kreiss, J. Oliger, Time dependent problems and difference methods, Pure and applied mathematics, J. Wiley, New York, Chichester, Brisbane, 1995. doi:10.1002/9781118548448.

[12] M. H. Carpenter, D. Gottlieb, S. Abarbanel, Time-stable boundary conditions for finite-difference schemes solving hyperbolic systems: methodology and application to high-order compact schemes, J. Comput. Phys. 111 (2) (1994) 220-236. doi:http://dx.doi.org/10.1006/jcph.1994.1057.

[13] J. Nordström, K. Mattsson, C. Swanson, Boundary conditions for a divergence free velocity-pressure formulation of the NavierStokes equations, J. Comput. Phys. 225 (1) (2007) 874-890. doi:10.1016/j.jcp.2007.01.010.

[14] D. Xiu, G. E. Karniadakis, The Wiener-Askey polynomial chaos for stochastic differential equations, SIAM J. Sci. Comput. 24 (2) (2002) 619-644. doi:http://dx.doi.org/10.1137/S1064827501387826.

[15] R. Askey, J. A. Wilson, Some Basic Hypergeometric Orthogonal Polynomials That Generalize Jacobi Polynomials, no. 319 in Memoirs of the American Mathematical Society, American Mathematical Society, 1985.

[16] B. K. Alpert, A class of bases in $L_{2}$ for the sparse representations of integral operators, SIAM J. Math. Anal. 24 (1993) 246-262. doi:10.1137/0524016. 
[17] X. Wan, G. E. Karniadakis, An adaptive multi-element generalized polynomial chaos method for stochastic differential equations, J. Comput. Phys. 209 (2005) 617-642. doi:10.1016/j.jcp.2005.03.023.

[18] P. Pettersson, A. Doostan, J. Nordström, On stability and monotonicity requirements of finite difference approximations of stochastic conservation laws with random viscosity, Comput. Method. Appl. M. 258 (2013) 134-151. doi:10.1016/j.cma.2013.02.009.

[19] G. Strang, Accurate partial difference methods, Numerische Mathematik 6 (1) (1964) 37-46. doi:10.1007/BF01386051.

[20] H.-O. Kreiss, J. Lorenz, Initial-Boundary Value Problems and the Navier-Stokes Equation, Classics in Applied Mathematics, Society for Industrial and Applied Mathematics, 2004. doi:http://dx.doi.org/10.1137/1.9780898719130.

[21] J. Silvester, Determinants of block matrices, Math. Gaz. 84 (501) (2000) 460-467. doi:10.2307/3620776.

[22] D. Gottlieb, D. Xiu, Galerkin method for wave equations with uncertain coefficients, Comm. Comput. Phys. 3 (2) (2008) 505-518.

[23] W. D. Henshaw, A fourth-order accurate method for the incompressible Navier-Stokes equations on overlapping grids, J. Comput. Phys. 113 (1) (1994) 13 - 25. doi:http://dx.doi.org/10.1006/jcph.1994.1114.

[24] N. A. Petersson, Stability of pressure boundary conditions for Stokes and Navier-Stokes equations, J. Comput. Phys. 172 (1) (2001) $40-70$. doi:http://dx.doi.org/10.1006/jcph.2001.6754.

[25] M. Svärd, On coordinate transformations for summation-by-parts operators, J. Sci. Comput. 20 (1) (2004) 29-42. doi:10.1023/A:1025881528802.

[26] M. H. Carpenter, J. Nordström, D. Gottlieb, A stable and conservative interface treatment of arbitrary spatial accuracy, J. Comput. Phys. 148 (1999) 341-365. doi:10.1006/jcph.1998.6114.

[27] K. Mattsson, J. Nordström, Summation by parts operators for finite difference approximations of second derivatives, J. Comput. Phys. 199 (2) (2004) 503-540. doi:10.1016/j.jcp.2004.03.001. 
[28] K. Mattsson, Summation by parts operators for finite difference approximations of second-derivatives with variable coefficients, J. Sci. Comput. 51 (2012) 650-682. doi:10.1007/s10915-011-9525-z.

[29] K. Mattsson, F. Ham, G. Iaccarino, Stable boundary treatment for the wave equation on second-order form, J. Sci. Comput. 41 (3) (2009) 366383. doi:10.1007/s10915-009-9305-1.

[30] M. Svärd, J. Nordström, On the order of accuracy for difference approximations of initial-boundary value problems, J. Comput. Phys. 218 (1) (2006) 333-352. doi:10.1016/j.jcp.2006.02.014.

[31] P. J. Roache, Verification of codes and calculations, AIAA Journal 36 (5) (1988) 696-702. doi:10.2514/2.457.

[32] K. Salari, P. Knupp, Code verification by the method of manufactured solutions, Tech. Rep. SAND 2000-1444, Sandia National Laboratories, Albuquerque, NM (2000). 